\title{
Health Care Spending in the United States and Other High-Income Countries
}

Irene Papanicolas, PhD; Liana R. Woskie, MSc; Ashish K. Jha, MD, MPH

IMPORTANCE Health care spending in the United States is a major concern and is higher than in other high-income countries, but there is little evidence that efforts to reform US health care delivery have had a meaningful influence on controlling health care spending and costs.

OBJECTIVE To compare potential drivers of spending, such as structural capacity and utilization, in the United States with those of 10 of the highest-income countries (United Kingdom, Canada, Germany, Australia, Japan, Sweden, France, the Netherlands, Switzerland, and Denmark) to gain insight into what the United States can learn from these nations.

EVIDENCE Analysis of data primarily from 2013-2016 from key international organizations including the Organisation for Economic Co-operation and Development (OECD), comparing underlying differences in structural features, types of health care and social spending, and performance between the United States and 10 high-income countries. When data were not available for a given country or more accurate country-level estimates were available from sources other than the OECD, country-specific data sources were used.

FINDINGS In 2016, the US spent $17.8 \%$ of its gross domestic product on health care, and spending in the other countries ranged from 9.6\% (Australia) to 12.4\% (Switzerland). The proportion of the population with health insurance was $90 \%$ in the US, lower than the other countries (range, 99\%-100\%), and the US had the highest proportion of private health insurance (55.3\%). For some determinants of health such as smoking, the US ranked second lowest of the countries ( $11.4 \%$ of the US population $\geq 15$ years smokes daily; mean of all 11 countries, $16.6 \%$ ), but the US had the highest percentage of adults who were overweight or obese at $70.1 \%$ (range for other countries, $23.8 \%-63.4 \%$; mean of all 11 countries, $55.6 \%$ ). Life expectancy in the US was the lowest of the 11 countries at 78.8 years (range for other countries, 80.7-83.9 years; mean of all 11 countries, 81.7 years), and infant mortality was the highest ( 5.8 deaths per 1000 live births in the US; 3.6 per 1000 for all 11 countries). The US did not differ substantially from the other countries in physician workforce (2.6 physicians per $1000 ; 43 \%$ primary care physicians), or nursing workforce (11.1 nurses per 1000). The US had comparable numbers of hospital beds (2.8 per 1000) but higher utilization of magnetic resonance imaging (118 per 1000) and computed tomography (245 per 1000) vs other countries. The US had similar rates of utilization (US discharges per 100000 were 192 for acute myocardial infarction, 365 for pneumonia, 230 for chronic obstructive pulmonary disease; procedures per 100000 were 204 for hip replacement, 226 for knee replacement, and 79 for coronary artery bypass graft surgery). Administrative costs of care (activities relating to planning, regulating, and managing health systems and services) accounted for $8 \%$ in the US vs a range of $1 \%$ to $3 \%$ in the other countries. For pharmaceutical costs, spending per capita was $\$ 1443$ in the US vs a range of $\$ 466$ to $\$ 939$ in other countries. Salaries of physicians and nurses were higher in the US; for example, generalist physicians salaries were $\$ 218173$ in the US compared with a range of $\$ 86607$ to $\$ 154126$ in the other countries.

CONCLUSIONS AND RELEVANCE The United States spent approximately twice as much as other high-income countries on medical care, yet utilization rates in the United States were largely similar to those in other nations. Prices of labor and goods, including pharmaceuticals, and administrative costs appeared to be the major drivers of the difference in overall cost between the United States and other high-income countries. As patients, physicians, policy makers, and legislators actively debate the future of the US health system, data such as these are needed to inform policy decisions.

JAMA. 2018;319(10):1024-1039. doi:10.1001/jama.2018.1150 $\leftarrow$ Viewpoint page 977 and Editorials pages 983,986 , 988, and 990

Animated Summary Video

Supplemental content and Audio

$+$

CME Quiz at

jamanetwork.com/learning
Author Affiliations: Department of Health Policy and Management, Harvard T. H. Chan School of Public Health, Boston, Massachusetts (Papanicolas, Woskie, Jha); Harvard Global Health Institute, Cambridge, Massachusetts (Papanicolas, Woskie Jha); Department of Health Policy, London School of Economics and Political Science, London, England (Papanicolas, Woskie).

Corresponding Author: Irene Papanicolas, PhD, Department of Health Policy, London School of Economics and Political Science, Houghton Street, London WC2A 2AE, England (i.n.papanicolas @lse.ac.uk). 
$\mathrm{T}$ he United States spends more per capita on health care than any other nation, substantially outpacing even other very high-income countries., ${ }^{1,2}$ However, despite its higher spending, the United States performs poorly in areas such as health care coverage and health outcomes. ${ }^{3-5}$ Higher spending without commensurate improved health outcomes at the population level has been a strong impetus for health care reform in the United States. ${ }^{6}$

Although it is well known that the United States spends more on health care than other countries, less is known about what explains these differences. The consensus has been that the US fee-for-service system is a primary factor, ${ }^{7}$ leading to fragmentation, overuse, and

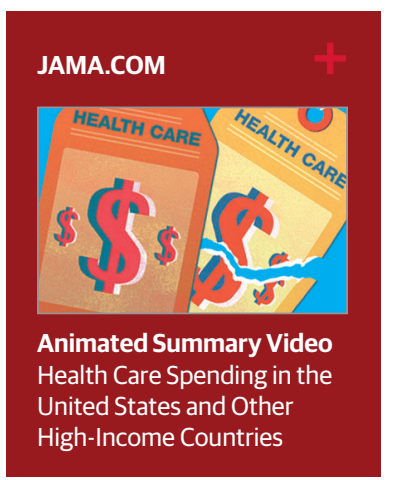
an underinvestment in social determinants of health, ${ }^{8-10}$ driving high utilization of health care services and poor outcomes. Older studies have found that the United States may underinvest in social services, ${ }^{11}$ although other data suggest that higher prices in the United States, especially for pharmaceuticals, may be a contributor to spending differences. ${ }^{12,13}$ One study suggested that increasing rates of outpatient spending and remuneration of clinicians is a major contributor to the cost difference between the United States and other countries. ${ }^{14}$ Given that other high-income countries are able to spend less and achieve better health outcomes, a more nuanced, data-driven understanding of all aspects of health care cost are needed to assist in reform of the US health care system.

The Organisation for Economic Co-operation and Development (OECD) and the Commonwealth Fund have recently collected and made available increasingly comparable data on inputs and performance of the health care systems across high-income countries. Using these and related data, we compared performance of the United States with 10 other high-income countries on key metrics that underpin health care spending. By examining granular data, we sought to understand why US health care costs are so much higher and where policy makers might target their efforts to encourage a more efficient system.

\section{Methods}

\section{Selection of Comparison Countries}

Ten high-income countries were selected for comparison. These countries were chosen because they were among the highestincome countries in the world, had relatively high health care spending, and had populations with similar demographic characteristics that have similar burdens of illness. ${ }^{3,15}$ Based on these criteria, the United Kingdom (consisting of England, Scotland, Wales, and Northern Ireland), Canada, Germany, Australia, Japan, Sweden, France, Denmark, the Netherlands, and Switzerland were chosen for comparison. These 10 selected countries represent different geographic areas and diverse health system structures.

\section{Key Points}

Question Why is health care spending in the United States so much greater than in other high-income countries?

Findings In 2016, the United States spent nearly twice as much as 10 high-income countries on medical care and performed less well on many population health outcomes. Contrary to some explanations for high spending, social spending and health care utilization in the United States did not differ substantially from other high-income nations. Prices of labor and goods, including pharmaceuticals and devices, and administrative costs appeared to be the main drivers of the differences in spending.

Meaning Efforts targeting utilization alone are unlikely to reduce the growth in health care spending in the United States; a more concerted effort to reduce prices and administrative costs is likely needed.

\section{Conceptual Framework and Indicator Selection}

To better understand the higher US health care costs relative to other high-income countries, a range of outcomes were explored. We first analyzed comparative data on general health system spending, including spending by function. Next, comparative inputs, including labor costs and structural capacity (which, aside from contributing to direct costs, may also influence maintenance costs or influence the price to use equipment) were examined. Because many of the leading explanations relating to higher health care costs involve the transformation of health care dollars to health care outcomes, ${ }^{16}$ we extended the analysis to examine a range of intermediate outputs-namely, access, utilization (inpatient, outpatient, major procedures), pharmaceutical spending and utilization, patient experience, and quality of care-as well as valued health system outcomes, such as population health. To provide a broader context of overall factors that can contribute to differences in health care spending, we also examined social spending, as well as demographic differences, risk factors, and prevalence of disease. In line with previous international comparisons, the health care system included all groups whose primary intent is to improve health. ${ }^{5,17}$

This approach resulted in the presentation of a total of 98 indicators across 7 domains: (1) general spending; (2) population health; (3) structural capacity; (4) utilization; (5) pharmaceuticals; (6) access and quality; and (7) equity. In each domain, measures were selected that were available across the majority of the countries in the analysis. We were unable to find comparable pricing data for most areas, such as for diagnostic procedures and treatments, except for workforce remuneration and pharmaceuticals. In the area of quality, the focus was on indicators that captured quality of prevention, primary care, and inpatient care across the areas of appropriateness, effectiveness, experience, and safety. In the area of access to care, variations related to financial costs as well as waiting times were explored. In addition, reflecting equity, variations related to service availability, quality of care, and cost were assessed.

\section{Data Sources}

Data were extracted from a range of databases compiled by international organizations, with the majority coming from the OECD. 


\begin{tabular}{|c|c|c|c|c|c|c|c|c|c|c|c|c|}
\hline \multicolumn{13}{|l|}{ Figure 1. Spending } \\
\hline Rank (highest to lowest) & 1 & 2 & 3 & 4 & 5 & 6 & 7 & 8 & 9 & 10 & 11 & Mean \\
\hline \multicolumn{13}{|l|}{ General } \\
\hline Overall population (in millions) & $\begin{array}{l}\text { US } \\
323\end{array}$ & $\begin{array}{l}\text { Japan } \\
127\end{array}$ & $\begin{array}{l}\text { Germany } \\
83\end{array}$ & $\begin{array}{l}\text { UK } \\
66\end{array}$ & $\begin{array}{l}\text { France } \\
64\end{array}$ & $\begin{array}{l}\text { Canada } \\
36\end{array}$ & $\begin{array}{l}\text { Australia } \\
24\end{array}$ & $\begin{array}{l}\text { NLD } \\
17\end{array}$ & $\begin{array}{l}\text { Sweden } \\
10\end{array}$ & $\begin{array}{l}\text { CHE } \\
8\end{array}$ & $\begin{array}{l}\text { Denmark } \\
6\end{array}$ & 69 \\
\hline Population $\geq 65$ y, \% & $\begin{array}{l}\text { Japan } \\
25.1\end{array}$ & $\begin{array}{l}\text { Germany } \\
21.4\end{array}$ & $\begin{array}{l}\text { Sweden } \\
19.9\end{array}$ & $\begin{array}{l}\text { France } \\
18.2\end{array}$ & $\begin{array}{l}\text { Denmark } \\
18.1\end{array}$ & $\begin{array}{l}\text { CHE } \\
17.5\end{array}$ & $\begin{array}{l}\text { UK } \\
17.3\end{array}$ & $\begin{array}{l}\text { NLD } \\
17.3\end{array}$ & $\begin{array}{l}\text { Canada } \\
15.7\end{array}$ & $\begin{array}{l}\text { Australia } \\
14.7\end{array}$ & $\begin{array}{l}\text { US } \\
14.5\end{array}$ & 18.2 \\
\hline $\begin{array}{l}\text { GDP per capita, US } \$ \\
\text { (in thousands) }\end{array}$ & $\begin{array}{l}\text { CHE } \\
54.00\end{array}$ & $\begin{array}{l}\text { Denmark } \\
53.40\end{array}$ & $\begin{array}{l}\text { US } \\
52.10\end{array}$ & $\begin{array}{l}\text { Sweden } \\
51.60\end{array}$ & $\begin{array}{l}\text { NLD } \\
46.30\end{array}$ & $\begin{array}{l}\text { Australia } \\
45.10\end{array}$ & $\begin{array}{l}\text { Germany } \\
42.90\end{array}$ & $\begin{array}{l}\text { Canada } \\
42.40\end{array}$ & $\begin{array}{l}\text { France } \\
41.00\end{array}$ & $\begin{array}{l}\text { UK } \\
38.50\end{array}$ & $\begin{array}{l}\text { Japan } \\
37.50\end{array}$ & 45.90 \\
\hline Land area (× $1000 \mathrm{sq} \mathrm{km)}$ & $\begin{array}{l}\text { Canada } \\
9985\end{array}$ & $\begin{array}{l}\text { US } \\
9834\end{array}$ & $\begin{array}{l}\text { Australia } \\
7741\end{array}$ & $\begin{array}{l}\text { France } \\
549\end{array}$ & $\begin{array}{l}\text { Sweden } \\
450\end{array}$ & $\begin{array}{l}\text { Japan } \\
378\end{array}$ & $\begin{array}{l}\text { Germany } \\
357\end{array}$ & $\begin{array}{l}\text { UK } \\
244\end{array}$ & $\begin{array}{l}\text { Denmark } \\
43\end{array}$ & $\begin{array}{l}\text { NLD } \\
42\end{array}$ & $\begin{array}{l}\text { CHE } \\
42\end{array}$ & 2697 \\
\hline $\begin{array}{l}\text { Poverty rate, } \% \text { below poverty } \\
\text { line of } 60 \%\end{array}$ & $\begin{array}{l}\text { US } \\
24\end{array}$ & $\begin{array}{l}\text { Japan } \\
22\end{array}$ & $\begin{array}{l}\text { Canada } \\
21\end{array}$ & $\begin{array}{l}\text { Australia } \\
20\end{array}$ & $\begin{array}{l}\text { UK } \\
18\end{array}$ & $\begin{array}{l}\text { Sweden } \\
17\end{array}$ & $\begin{array}{l}\text { CHE } \\
17\end{array}$ & $\begin{array}{l}\text { Germany } \\
16\end{array}$ & $\begin{array}{l}\text { France } \\
15\end{array}$ & $\begin{array}{l}\text { NLD } \\
15\end{array}$ & $\begin{array}{l}\text { Denmark } \\
12\end{array}$ & 18 \\
\hline \multicolumn{13}{|l|}{ Health spending } \\
\hline $\begin{array}{l}\text { Total spending on health, } \\
\% \text { of total national GDP }\end{array}$ & $\begin{array}{l}\text { US } \\
17.8\end{array}$ & $\begin{array}{l}\text { CHE } \\
12.4\end{array}$ & $\begin{array}{l}\text { Sweden } \\
11.9\end{array}$ & $\begin{array}{l}\text { Germany } \\
11.3\end{array}$ & $\begin{array}{l}\text { France } \\
11\end{array}$ & $\begin{array}{l}\text { Japan } \\
10.9\end{array}$ & $\begin{array}{l}\text { Denmark } \\
10.8\end{array}$ & $\begin{array}{l}\text { NLD } \\
10.5\end{array}$ & $\begin{array}{l}\text { Canada } \\
10.3\end{array}$ & $\begin{array}{l}\text { UK } \\
9.7\end{array}$ & $\begin{array}{l}\text { Australia } \\
9.6\end{array}$ & 11.5 \\
\hline $\begin{array}{l}\text { Public spending on health, } \\
\% \text { of total national GDP }\end{array}$ & $\begin{array}{l}\text { Sweden } \\
10\end{array}$ & $\begin{array}{l}\text { NLD } \\
9.5\end{array}$ & $\begin{array}{l}\text { Denmark } \\
9.2\end{array}$ & $\begin{array}{l}\text { Germany } \\
8.7\end{array}$ & $\begin{array}{l}\text { France } \\
8.7\end{array}$ & $\begin{array}{l}\text { Japan } \\
8.6\end{array}$ & $\begin{array}{l}\text { US } \\
8.3\end{array}$ & $\begin{array}{l}\text { CHE } \\
7.7\end{array}$ & $\begin{array}{l}\text { UK } \\
7.6\end{array}$ & $\begin{array}{l}\text { Canada } \\
7.4\end{array}$ & $\begin{array}{l}\text { Australia } \\
6.3\end{array}$ & 8.4 \\
\hline $\begin{array}{l}\text { Mean spending on health } \\
\text { per capita, US } \$\end{array}$ & $\begin{array}{l}\text { US } \\
9403\end{array}$ & $\begin{array}{l}\text { Sweden } \\
6808\end{array}$ & $\begin{array}{l}\text { CHE } \\
6787\end{array}$ & $\begin{array}{l}\text { Denmark } \\
6463\end{array}$ & $\begin{array}{l}\text { NLD } \\
5202\end{array}$ & $\begin{array}{l}\text { Germany } \\
5182\end{array}$ & $\begin{array}{l}\text { Canada } \\
4641\end{array}$ & $\begin{array}{l}\text { Australia } \\
4357\end{array}$ & $\begin{array}{l}\text { Japan } \\
3727\end{array}$ & $\begin{array}{l}\text { France } \\
3661\end{array}$ & $\begin{array}{l}\text { UK } \\
3377\end{array}$ & 5419 \\
\hline \multicolumn{13}{|c|}{ Health expenditure by function of care as a $\%$ of total national health expenditure } \\
\hline Inpatient care & $\begin{array}{l}\text { NLD } \\
32\end{array}$ & $\begin{array}{l}\text { Australia } \\
31\end{array}$ & $\begin{array}{l}\text { France } \\
30\end{array}$ & $\begin{array}{l}\text { CHE } \\
28\end{array}$ & $\begin{array}{l}\text { Denmark } \\
28\end{array}$ & $\begin{array}{l}\text { Germany } \\
27\end{array}$ & $\begin{array}{l}\text { Japan } \\
27\end{array}$ & $\begin{array}{l}\text { UK } \\
24\end{array}$ & $\begin{array}{l}\text { Sweden } \\
21\end{array}$ & $\begin{array}{l}\text { US } \\
19\end{array}$ & $\begin{array}{l}\text { Canada } \\
17\end{array}$ & 26 \\
\hline Outpatient care & $\begin{array}{l}\text { US } \\
42\end{array}$ & $\begin{array}{l}\text { Australia } \\
39\end{array}$ & $\begin{array}{l}\text { Canada } \\
36\end{array}$ & $\begin{array}{l}\text { Denmark } \\
34\end{array}$ & $\begin{array}{l}\text { CHE } \\
33\end{array}$ & $\begin{array}{l}\text { Sweden } \\
31\end{array}$ & $\begin{array}{l}\text { UK } \\
30\end{array}$ & $\begin{array}{l}\text { Japan } \\
27\end{array}$ & $\begin{array}{l}\text { Germany } \\
23\end{array}$ & $\begin{array}{l}\text { France } \\
23\end{array}$ & $\begin{array}{l}\text { NLD } \\
22\end{array}$ & 31 \\
\hline Long-term care & $\begin{array}{l}\text { Sweden } \\
26\end{array}$ & $\begin{array}{l}\text { NLD } \\
26\end{array}$ & $\begin{array}{l}\text { Denmark } \\
24\end{array}$ & $\begin{array}{l}\text { CHE } \\
19\end{array}$ & $\begin{array}{l}\text { Japan } \\
19\end{array}$ & $\begin{array}{l}\text { UK } \\
18\end{array}$ & $\begin{array}{l}\text { Germany } \\
16\end{array}$ & $\begin{array}{l}\text { Canada } \\
14\end{array}$ & $\begin{array}{l}\text { France } \\
11\end{array}$ & $\begin{array}{l}\text { US } \\
5\end{array}$ & $\begin{array}{l}\text { Australia } \\
2\end{array}$ & 16 \\
\hline Medical goods & $\begin{array}{l}\text { Germany } \\
20\end{array}$ & $\begin{array}{l}\text { France } \\
20\end{array}$ & $\begin{array}{l}\text { Canada } \\
20\end{array}$ & $\begin{array}{l}\text { Japan } \\
20\end{array}$ & $\begin{array}{l}\text { Australia } \\
17\end{array}$ & $\begin{array}{l}\text { UK } \\
15\end{array}$ & $\begin{array}{l}\text { US } \\
14\end{array}$ & $\begin{array}{l}\text { CHE } \\
13\end{array}$ & $\begin{array}{l}\text { Sweden } \\
12\end{array}$ & $\begin{array}{l}\text { NLD } \\
12\end{array}$ & $\begin{array}{l}\text { Denmark } \\
10\end{array}$ & 16 \\
\hline $\begin{array}{l}\text { Governance and } \\
\text { administration }\end{array}$ & $\begin{array}{l}\text { US } \\
8\end{array}$ & $\begin{array}{l}\text { Germany } \\
5\end{array}$ & $\begin{array}{l}\text { NLD } \\
4\end{array}$ & $\begin{array}{l}\text { CHE } \\
4\end{array}$ & $\begin{array}{l}\text { Canada } \\
3\end{array}$ & $\begin{array}{l}\text { Australia } \\
3\end{array}$ & $\begin{array}{l}\text { UK } \\
2\end{array}$ & $\begin{array}{l}\text { Sweden } \\
2\end{array}$ & $\begin{array}{l}\text { Denmark } \\
2\end{array}$ & $\begin{array}{l}\text { France } \\
1\end{array}$ & $\begin{array}{l}\text { Japan } \\
1\end{array}$ & 3 \\
\hline Home-based care & $\begin{array}{l}\text { France } \\
4\end{array}$ & $\begin{array}{l}\text { US } \\
3\end{array}$ & $\begin{array}{l}\text { UK } \\
3\end{array}$ & $\begin{array}{l}\text { Japan } \\
3\end{array}$ & $\begin{array}{l}\text { Germany } \\
1\end{array}$ & $\begin{array}{l}\text { Sweden } \\
0\end{array}$ & $\begin{array}{l}\text { NLD } \\
0\end{array}$ & $\begin{array}{l}\text { Canada } \\
0\end{array}$ & $\begin{array}{l}\text { Australia } \\
0\end{array}$ & $\begin{array}{l}\text { CHE } \\
\text { NA }\end{array}$ & $\begin{array}{l}\text { Denmark } \\
\text { NA }\end{array}$ & 2 \\
\hline Preventive care & $\begin{array}{l}\text { Canada } \\
6\end{array}$ & $\begin{array}{l}\text { UK } \\
5\end{array}$ & $\begin{array}{l}\text { NLD } \\
4\end{array}$ & $\begin{array}{l}\text { US } \\
3\end{array}$ & $\begin{array}{l}\text { Germany } \\
3\end{array}$ & $\begin{array}{l}\text { Sweden } \\
3\end{array}$ & $\begin{array}{l}\text { Denmark } \\
3\end{array}$ & $\begin{array}{l}\text { Japan } \\
3\end{array}$ & $\begin{array}{l}\text { France } \\
2\end{array}$ & $\begin{array}{l}\text { CHE } \\
2\end{array}$ & $\begin{array}{l}\text { Australia } \\
2\end{array}$ & 3 \\
\hline Other & $\begin{array}{l}\text { France } \\
9\end{array}$ & $\begin{array}{l}\text { US } \\
6\end{array}$ & $\begin{array}{l}\text { Australia } \\
6\end{array}$ & $\begin{array}{l}\text { Germany } \\
5\end{array}$ & $\begin{array}{l}\text { Sweden } \\
5\end{array}$ & $\begin{array}{l}\text { Canada } \\
4\end{array}$ & $\begin{array}{l}\text { UK } \\
3\end{array}$ & $\begin{array}{l}\text { CHE } \\
1\end{array}$ & $\begin{array}{l}\text { Japan } \\
1\end{array}$ & $\begin{array}{l}\text { NLD } \\
0\end{array}$ & $\begin{array}{l}\text { Denmark } \\
0\end{array}$ & 4 \\
\hline $\begin{array}{l}\text { Population with health care } \\
\text { coverage, } \%\end{array}$ & $\begin{array}{l}\text { UK } \\
100\end{array}$ & $\begin{array}{l}\text { Sweden } \\
100\end{array}$ & $\begin{array}{l}\text { CHE } \\
100\end{array}$ & $\begin{array}{l}\text { Denmark } \\
100\end{array}$ & $\begin{array}{l}\text { Canada } \\
100\end{array}$ & $\begin{array}{l}\text { Japan } \\
100\end{array}$ & $\begin{array}{l}\text { Australia } \\
100\end{array}$ & $\begin{array}{l}\text { France } \\
99.9\end{array}$ & $\begin{array}{l}\text { NLD } \\
99.9\end{array}$ & $\begin{array}{l}\text { Germany } \\
99.8\end{array}$ & $\begin{array}{l}\text { US } \\
90\end{array}$ & 99 \\
\hline
\end{tabular}

GDP indicates gross domestic product; NA, not applicable. CHE indicates Switzerland; NLD, the Netherlands. See eTable 1 in Supplement 2 for data ordered by country.

Data on structural equipment, workforce, utilization, pharmaceutical spending, access, and quality were accessed from OECD.stat and the OECD 2015 Health Care at a Glance report. Additional data on health spending, health system, and country characteristics were obtained from the World Bank International Bank for Reconstruction and Development-International Development Association database and the 2016 OECD Health Systems Characteristics Survey.

Data on retail pharmaceutical spending per capita were obtained from the OECD for all countries. Data on total pharmaceutical spending per capita were obtained from Intercontinental Marketing Services or the International Federation of Pharmaceutical Manufacturers and Associations. Pharmaceutical data on country-level output of new chemical entities was taken from Daemmrich. ${ }^{18}$ Population perceptions of the health system and select access measures were obtained from the 2016 Commonwealth Fund Survey of Consumers. ${ }^{15}$
All data on per capita spending, gross domestic product (GDP), and remuneration were translated into US dollar equivalents, with exchange rates based on purchasing power parities of national currencies. Remuneration data were then converted to 2017 dollars using the US Consumer Price Index in line with Laugesen and Glied. ${ }^{19}$ Data on health spending are presented by function of care as a percentage of the country's total spending on health consistent with System of Health Accounts categorization, with adaptations for outpatient spending to address issues of comparability with the United States' National Health Expenditure Accounts (eTable 2 in Supplement 1). When OECD data were not available for a given country or more accurate country-level estimates were available, country-specific data sources were used. The focus was on indicators from 2013 onward with an occasional exception. For example, for the United States, data for the horizontal index, neonatal mortality by low birth weight, and antibiotic prescribing were from 2009, 2004, and 2004, respectively. 
Figure 2. Health Spending as a Percentage of Gross Domestic Product

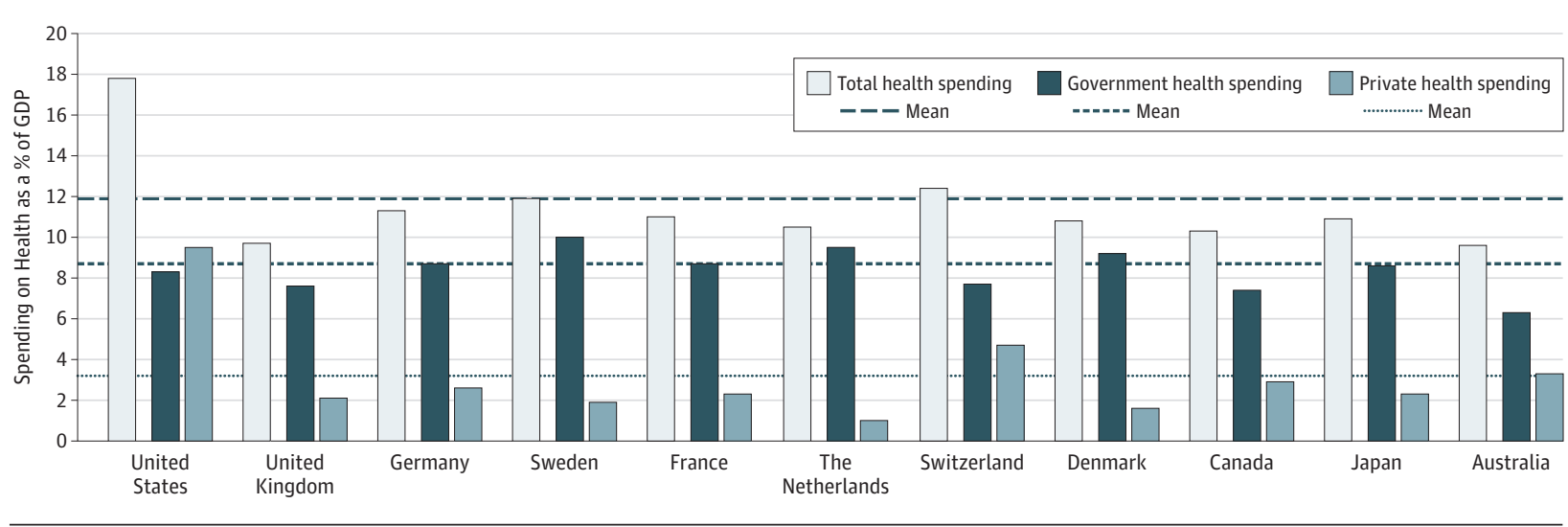

Supplement 1 includes tables that provide a breakdown of sources and methods for the data reported herein. In these tables, we note issues of comparability and timeliness for each indicator, such as workforce. In figures describing data for each of the 7 domains, a simple mean of the data for each indicator across all 11 countries is presented in the final column. Throughout the Results section in the text, all comparative findings are presented descriptively.

\section{Results}

Demographic Characteristics and Health Care Spending In 2016, the US population was significantly larger than all comparison countries at 323 million (Figure 1 and eTable 1 in Supplement 2). Japan had the next largest population with 127 million. The US system also covered the second largest geographical area ( 9834000 sq km), following Canada (9 985000 sq km). The other countries other than Australia had much smaller land mass. In 2016, the United States spent $17.8 \%$ of its GDP on health care (range of the other countries, 9.6\%-12.4\%; mean of all 11 countries, $11.5 \%$ ) (Figure 1 and Figure 2) and had almost double the health spending per capita (mean, \$9403) compared with the other countries (range, \$3377-\$6808; mean of all 11 countries, \$5419). Although the United States spent more, the percentage of the population with health insurance in the United States was 90\%, lower than in all of the other countries (range, 99\%-100\%).

All systems had relatively similar levels of public spending as a percentage of GDP (defined as spending from government and/or social or compulsory insurance funds), with the United States spending at about the mean level (8.3\%) of all the countries, although, unlike the other countries, this spending covered only about $37 \%$ of the population. By expenditure as a function of care, the United States spent only $19 \%$ of its health spending on inpatient care, which excludes same-day hospital care. This proportion was less than that of all other countries, with Australia (31\%) and the Netherlands (32\%) spending the most (Figure 1). The United States spent a greater proportion than the other countries on outpatient care $(44 \%$ compared with a mean of $31 \%$ ) and governance and administration, which includes activities relating to planning, regulating, and managing health systems and services ( $8 \%$ compared with a mean of $3 \%$ ).
Across the 11 countries, the United States had the lowest percentage of the population older than 65 years ( $14.5 \%$ compared with a mean of $18.2 \%$ ) and also had the highest rate of poverty, with $24 \%$ of the population living below the poverty line, followed by Japan (22\%) and Canada (21\%). The United States ranked below the mean but was not an outlier with regard to total social spending (spending on old age, incapacity, labor market, education, family, and housing [Figure 3]) at $16.7 \%$ of GDP (compared with a mean of $19.4 \%$ of GDP in all 11 countries). This reflected public social spending, which was, at $11.3 \%$ of GDP, below the mean of all 11 countries (15.3\% of GDP). The United States ranked fourth with regard to private social spending at $5.4 \%$ of GDP (compared with a mean of $4.1 \%$ ) and was similar to the United Kingdom (5.6\%) and ranked behind the Netherlands (7.1\%) and Switzerland (6.0\%), reflecting mostly private pension payments. ${ }^{20}$

\section{Insurance System Characteristics}

The structural characteristics of the health care system are detailed in the Table. Three countries, the United Kingdom, Sweden, and Denmark, have national health care systems, whereas Canada and Australia have regionally administered universal insurance programs. Germany, France, the Netherlands, and Switzerland have statutory/mandatory health insurance systems. Only the United States has a voluntary, private employer-based and individual-based system. All of the countries except the United States have an automatic or compulsory enrollment process. Private insurance as the primary form of insurance is highest in the United States at $55.3 \%$, followed by Germany at $10.8 \%$. The majority of the countries do not have private insurance as the primary form of insurance.

\section{Population Health}

Among important determinants of health, the United States had the highest percentage of overweight or obese adults $(70.1 \%$ compared with a mean of $55.6 \%$ ) but had relatively low smoking rates (11.4\% of the population compared with a mean of 16.6\%) (Figure 4 and eTable 2 in Supplement 2). The drinking rate (8.8 L per capita) and unemployment rate (4.4\%) in the United States are both close to the mean values of all 11 countries at $9.1 \mathrm{~L}$ per capita and $5.4 \%$, respectively. 


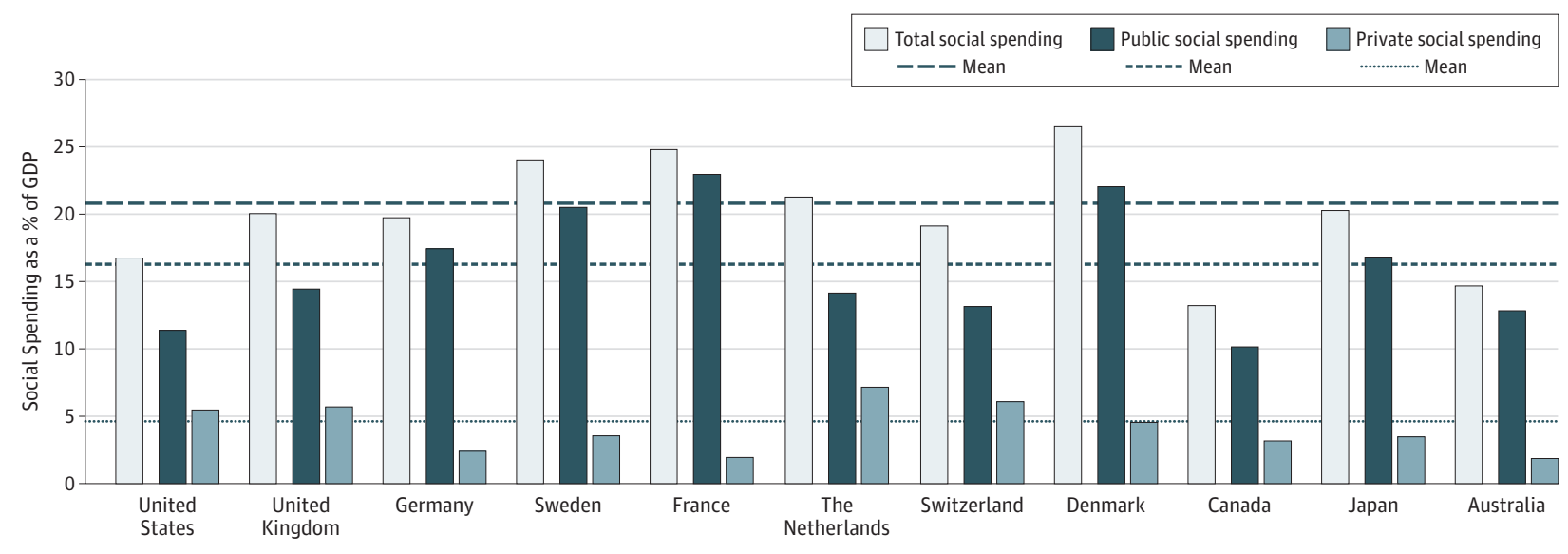

Social spending is the provision by public (and private) institutions of benefits to and financial contributions targeted at households and individuals to provide support during circumstances that adversely affect their welfare, provided that the provision of the benefits and financial contributions constitutes neither a direct payment for a particular good or service nor an individual contract or transfer. Such benefits can be cash transfers or can be direct (in-kind) provision of goods and services. Main spending areas include old age, health, family, incapacity, labor market, and housing (Organisation for Economic Co-operation and Development). Private social spending is functionally the same as public social spending but provided through a private mechanism. Social benefits delivered through the private sector (not transfers between individuals) involve an element of compulsion and/or interpersonal redistribution; for example, through pooling of contributions and risk sharing. This may include old-age pensions and support services for older adults, survivor benefits, disability and sickness cash benefits, family support, unemployment benefits, housing support (eg, rent subsidies), and other social policy areas excluding health spending. Pensions constitute an important part of private social spending in the United States and can be mandatory or voluntary. Independent, out-of-pocket spending on social services is not included.
The United States consistently had the poorest population health outcomes (Figure 4). The United States had the lowest life expectancy ( 78.8 years compared with a mean of 81.7 years) and the lowest health-adjusted life expectancy (69.1 years compared with a mean of 72.0 years). The variability of life expectancy across the United States (ranging from 81.3 years in Hawaii to 75 years in Mississippi) (eTable 3 in Supplement 1) was similar to that of the life expectancy across all countries in the study.

The United States had the highest infant mortality ( 5.8 deaths per 1000 live births compared with a mean of 3.6), neonatal mortality (4.0 deaths per 1000 live births compared with a mean of 2.6), and maternal mortality (26.4 deaths per 100000 live births compared with a mean of 8.4) (Figure 4). The United States also had the second highest percentage of infants with low birth weight $(8.1 \%$ compared with a mean of $6.6 \%$ ). Japan had the highest low birth weight (9.5\%). When adjusting neonatal mortality to exclude deaths of infants born weighing less than $1000 \mathrm{~g}$, the United States ranked fifth relative to the other countries, with 1.61 deaths per 1000 live births, compared with a mean of 1.70 for all 11 countries.

\section{Workforce and Structural Capacity}

The physician workforce in the United States was lower than the mean of all 11 countries at 2.6 per 1000 population compared with 3.3 per 1000 population (Figure 5 and eTable 3 in Supplement 2). The proportion of US physicians who were primary care physicians (43\%) was the same as the mean of all 11 countries. Using a functionality-based approach to identifying primary care physicians, US general internists provided a significant amount of primary care, whereas internists in Canada almost exclusively provided acute hospital-based care (Figure 6). Compared with countries with comparable data, mean remuneration of generalists, special- ists, and nurses was higher in the United States. When adjusting for purchasing power parity, the mean US remuneration for generalists was $\$ 218173$, nearly double the mean remuneration in all 11 countries, which ranged from $\$ 86607$ in Sweden to $\$ 154126$ in Germany. The remuneration for specialists was higher in the United States at $\$ 316000$ compared with other countries, ranging from $\$ 98452$ in Sweden to $\$ 202291$ in Australia). The remuneration of nurses was also higher in the United States (\$74 160) than in other countries, where it ranged from $\$ 42492$ in France to $\$ 65082$ in the Netherlands. The remuneration of health care professionals as a ratio to the mean national wage was highest in the United States for specialists (5.3 compared with a mean of 3.7), generalists (3.6 compared with a mean of 2.7$)$, and nurses (1.23 compared with a mean of 1.1).

There was notable variation between countries in the supply of medical equipment, such as magnetic resonance imaging (MRI) units, computed tomography (CT) machines, and mammography machines. Japan had the highest number of MRI units and CT scanners per population (approximately 52 and 107 per 1 million population, respectively), and the United States had the second highest for MRI (38 per 1 million population) and third highest for CT (41 per 1 million population). The lowest per capita rate for both MRI units and CT scanners was in the United Kingdom, with 7.2 MRI units and 9.5 CT scanners per 1 million population. The United States had fewer hospital beds per 1000 population (2.8) than Japan (13.2) and Germany (8.2) and fewer long-term beds per 1000 population older than 65 years (38.8) compared with the mean of the study countries (54.2).

\section{Utilization}

The United States' utilization of health care services was similar to the other countries (Figure 7 and eTable 4 in Supplement 2), 


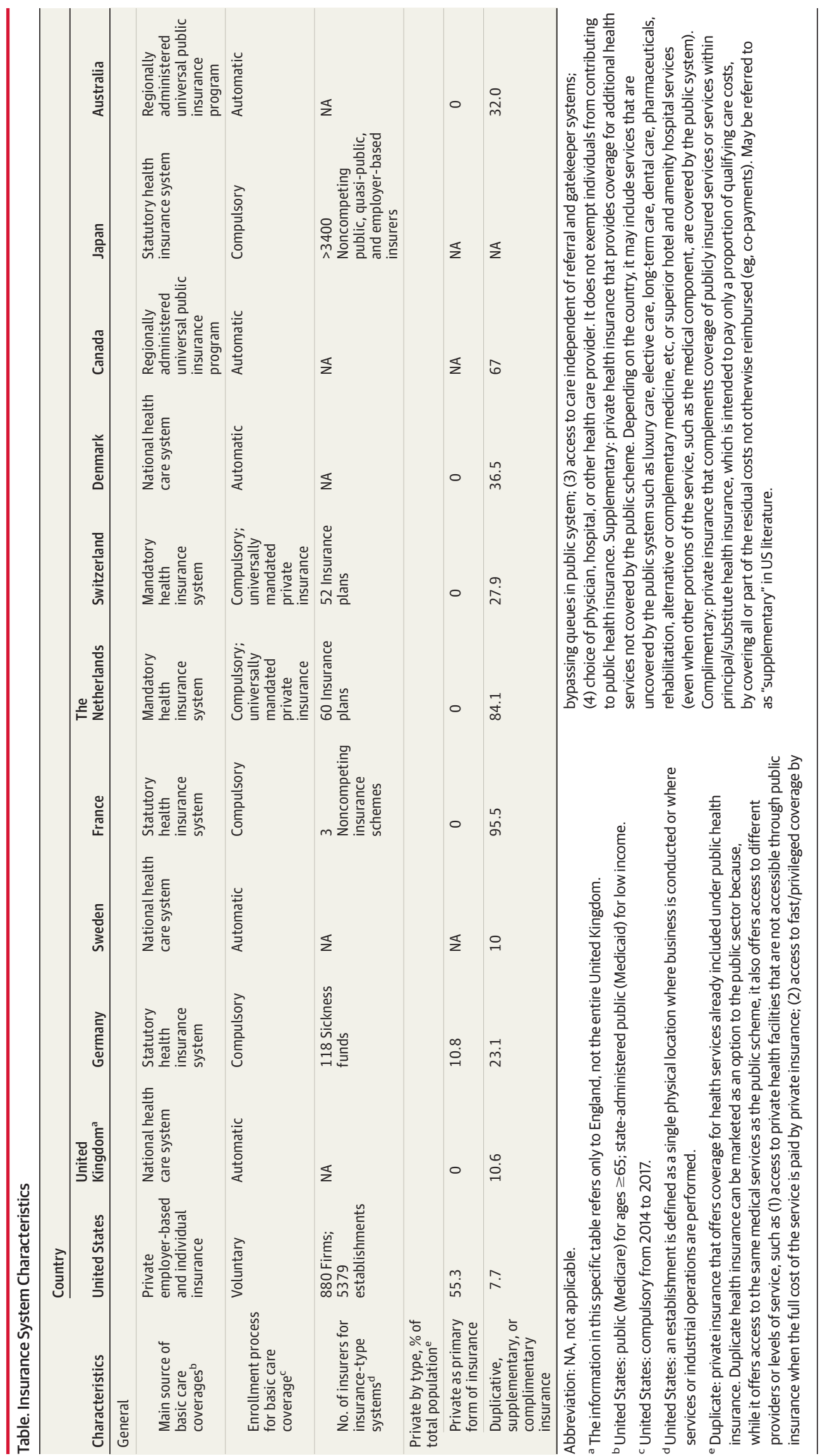




\begin{tabular}{|c|c|c|c|c|c|c|c|c|c|c|c|c|}
\hline Rank (highest to lowest) & 1 & 2 & 3 & 4 & 5 & 6 & 7 & 8 & 9 & 10 & 11 & Mean \\
\hline \multicolumn{13}{|l|}{ Determinants of health } \\
\hline $\begin{array}{l}\text { Smoking, } \% \text { of population } \\
\text { aged } \geq 15 \text { y who smoke daily }\end{array}$ & $\begin{array}{l}\text { France } \\
22.4\end{array}$ & $\begin{array}{l}\text { Germany } \\
20.9\end{array}$ & $\begin{array}{l}\text { CHE } \\
20.4\end{array}$ & $\begin{array}{l}\text { NLD } \\
19\end{array}$ & $\begin{array}{l}\text { Japan } \\
18.2\end{array}$ & $\begin{array}{l}\text { Denmark } \\
17\end{array}$ & $\begin{array}{l}\text { UK } \\
16.1\end{array}$ & $\begin{array}{l}\text { Canada } \\
14\end{array}$ & $\begin{array}{l}\text { Australia } \\
12.4\end{array}$ & $\begin{array}{l}\text { US } \\
11.4\end{array}$ & $\begin{array}{l}\text { Sweden } \\
11.2\end{array}$ & 16.6 \\
\hline $\begin{array}{l}\text { Alcohol consumption, } L \text { per } \\
\text { capita in population aged } \geq 15 \mathrm{y}\end{array}$ & $\begin{array}{l}\text { France } \\
11.9\end{array}$ & $\begin{array}{l}\text { Germany } \\
11\end{array}$ & $\begin{array}{l}\text { Australia } \\
9.7\end{array}$ & $\begin{array}{l}\text { UK } \\
9.5\end{array}$ & $\begin{array}{l}\text { CHE } \\
9.5\end{array}$ & $\begin{array}{l}\text { Denmark } \\
9.4\end{array}$ & $\begin{array}{l}\text { US } \\
8.8\end{array}$ & $\begin{array}{l}\text { Canada } \\
8.1\end{array}$ & $\begin{array}{l}\text { NLD } \\
8\end{array}$ & $\begin{array}{l}\text { Sweden } \\
7.2\end{array}$ & $\begin{array}{l}\text { Japan } \\
7.2\end{array}$ & 9.1 \\
\hline $\begin{array}{l}\text { Obese or overweight, } \% \text { of } \\
\text { population aged } \geq 15 y\end{array}$ & $\begin{array}{l}\text { US } \\
70.1\end{array}$ & $\begin{array}{l}\text { Australia } \\
63.4\end{array}$ & $\begin{array}{l}\text { UK } \\
62.9\end{array}$ & $\begin{array}{l}\text { Canada } \\
60.3\end{array}$ & $\begin{array}{l}\text { Germany } \\
60\end{array}$ & $\begin{array}{l}\text { France } \\
49\end{array}$ & $\begin{array}{l}\text { Sweden } \\
48.3^{a}\end{array}$ & $\begin{array}{l}\text { NLD } \\
47.4^{\mathrm{a}}\end{array}$ & $\begin{array}{l}\text { Denmark } \\
47.4^{\mathrm{a}}\end{array}$ & $\begin{array}{l}\text { CHE } \\
41^{a}\end{array}$ & $\begin{array}{l}\text { Japan } \\
23.8\end{array}$ & 55.6 \\
\hline \multicolumn{13}{|l|}{ Life expectancy } \\
\hline $\begin{array}{l}\text { Life expectancy in total } \\
\text { population at birth, mean, y }\end{array}$ & $\begin{array}{l}\text { Japan } \\
83.9\end{array}$ & $\begin{array}{l}\text { CHE } \\
83\end{array}$ & $\begin{array}{l}\text { Australia } \\
82.5\end{array}$ & $\begin{array}{l}\text { France } \\
82.4\end{array}$ & $\begin{array}{l}\text { Sweden } \\
82.3\end{array}$ & $\begin{array}{l}\text { Canada } \\
81.7\end{array}$ & $\begin{array}{l}\text { NLD } \\
81.6\end{array}$ & $\begin{array}{l}\text { UK } \\
81\end{array}$ & $\begin{array}{l}\text { Denmark } \\
80.8\end{array}$ & $\begin{array}{l}\text { Germany } \\
80.7\end{array}$ & $\begin{array}{l}\text { US } \\
78.8\end{array}$ & 81.7 \\
\hline $\begin{array}{l}\text { Health-adjusted life } \\
\text { expectancy, mean, y }\end{array}$ & $\begin{array}{l}\text { Japan } \\
74.9\end{array}$ & $\begin{array}{l}\text { CHE } \\
73.1\end{array}$ & $\begin{array}{l}\text { France } \\
72.6\end{array}$ & $\begin{array}{l}\text { Canada } \\
72.3\end{array}$ & $\begin{array}{l}\text { NLD } \\
72.2\end{array}$ & $\begin{array}{l}\text { Sweden } \\
72\end{array}$ & $\begin{array}{l}\text { Australia } \\
71.9\end{array}$ & $\begin{array}{l}\text { UK } \\
71.4\end{array}$ & $\begin{array}{l}\text { Germany } \\
71.3\end{array}$ & $\begin{array}{l}\text { Denmark } \\
71.2\end{array}$ & $\begin{array}{l}\text { US } \\
69.1\end{array}$ & 72 \\
\hline $\begin{array}{l}\text { Life expectancy for women } \\
\text { aged } \geq 40 y \text {, mean, } y\end{array}$ & $\begin{array}{l}\text { Japan } \\
47.7\end{array}$ & $\begin{array}{l}\text { France } \\
46.4\end{array}$ & $\begin{array}{l}\text { CHE } \\
45.8\end{array}$ & $\begin{array}{l}\text { Australia } \\
45.4\end{array}$ & $\begin{array}{l}\text { Sweden } \\
44.8\end{array}$ & $\begin{array}{l}\text { Canada } \\
44.8\end{array}$ & $\begin{array}{l}\text { Germany } \\
43.9\end{array}$ & $\begin{array}{l}\text { NLD } \\
43.9\end{array}$ & $\begin{array}{l}\text { UK } \\
43.7\end{array}$ & $\begin{array}{l}\text { Denmark } \\
43.4\end{array}$ & $\begin{array}{l}\text { US } \\
42.6\end{array}$ & 44.8 \\
\hline $\begin{array}{l}\text { Life expectancy for men } \\
\text { aged } \geq 40 \mathrm{y} \text {, mean, } \mathrm{y}\end{array}$ & $\begin{array}{l}\text { CHE } \\
42\end{array}$ & $\begin{array}{l}\text { Japan } \\
41.8\end{array}$ & $\begin{array}{l}\text { Australia } \\
41.7\end{array}$ & $\begin{array}{l}\text { Sweden } \\
41.5\end{array}$ & $\begin{array}{l}\text { Canada } \\
41.1\end{array}$ & $\begin{array}{l}\text { NLD } \\
40.8\end{array}$ & $\begin{array}{l}\text { France } \\
40.6\end{array}$ & $\begin{array}{l}\text { UK } \\
40.5\end{array}$ & $\begin{array}{l}\text { Denmark } \\
39.8\end{array}$ & $\begin{array}{l}\text { Germany } \\
39.4\end{array}$ & $\begin{array}{l}\text { US } \\
38.7\end{array}$ & 40.7 \\
\hline \multicolumn{13}{|l|}{ Maternal and infant health } \\
\hline $\begin{array}{l}\text { Maternal mortality, deaths per } \\
100000 \text { live births }\end{array}$ & $\begin{array}{l}\text { US } \\
26.4\end{array}$ & $\begin{array}{l}\text { UK } \\
9.2\end{array}$ & $\begin{array}{l}\text { Germany } \\
9\end{array}$ & $\begin{array}{l}\text { France } \\
7.8\end{array}$ & $\begin{array}{l}\text { Canada } \\
7.3\end{array}$ & $\begin{array}{l}\text { NLD } \\
6.7\end{array}$ & $\begin{array}{l}\text { Japan } \\
6.4\end{array}$ & $\begin{array}{l}\text { CHE } \\
5.8\end{array}$ & $\begin{array}{l}\text { Australia } \\
5.5\end{array}$ & $\begin{array}{l}\text { Sweden } \\
4.4\end{array}$ & $\begin{array}{l}\text { Denmark } \\
4.2\end{array}$ & 8.4 \\
\hline $\begin{array}{l}\text { Infant mortality, deaths per } \\
1000 \text { live births }\end{array}$ & $\begin{array}{l}\text { US } \\
5.8\end{array}$ & $\begin{array}{l}\text { Canada } \\
5.1\end{array}$ & $\begin{array}{l}\text { UK } \\
3.9\end{array}$ & $\begin{array}{l}\text { CHE } \\
3.9\end{array}$ & $\begin{array}{l}\text { France } \\
3.8\end{array}$ & $\begin{array}{l}\text { Denmark } \\
3.7\end{array}$ & $\begin{array}{l}\text { Germany } \\
3.3\end{array}$ & $\begin{array}{l}\text { Australia } \\
3.2\end{array}$ & $\begin{array}{l}\text { Sweden } \\
2.5\end{array}$ & $\begin{array}{l}\text { NLD } \\
2.5\end{array}$ & $\begin{array}{l}\text { Japan } \\
2.1\end{array}$ & 3.6 \\
\hline $\begin{array}{l}\text { Neonatal mortality, deaths per } \\
1000 \text { live births }\end{array}$ & $\begin{array}{l}\text { US } \\
4\end{array}$ & $\begin{array}{l}\text { Canada } \\
3.2\end{array}$ & $\begin{array}{l}\text { CHE } \\
3.1\end{array}$ & $\begin{array}{l}\text { Denmark } \\
3\end{array}$ & $\begin{array}{l}\text { UK } \\
2.7\end{array}$ & $\begin{array}{l}\text { France } \\
2.6\end{array}$ & $\begin{array}{l}\text { NLD } \\
2.5\end{array}$ & $\begin{array}{l}\text { Germany } \\
2.3\end{array}$ & $\begin{array}{l}\text { Australia } \\
2.3\end{array}$ & $\begin{array}{l}\text { Sweden } \\
1.7\end{array}$ & $\begin{array}{l}\text { Japan } \\
0.9\end{array}$ & 2.6 \\
\hline $\begin{array}{l}\text { Neonatal mortality, deaths per } \\
1000 \text { live births excluding }<1000 \mathrm{~g}\end{array}$ & $\begin{array}{l}\text { Denmark } \\
2.09\end{array}$ & $\begin{array}{l}\text { NLD } \\
1.96\end{array}$ & $\begin{array}{l}\text { UK } \\
1.77\end{array}$ & $\begin{array}{l}\text { Canada } \\
1.63\end{array}$ & $\begin{array}{l}\text { US } \\
1.61\end{array}$ & $\begin{array}{l}\text { Sweden } \\
1.56\end{array}$ & $\begin{array}{l}\text { Germany } \\
1.49\end{array}$ & $\begin{array}{l}\text { France } \\
\text { NA }\end{array}$ & $\begin{array}{l}\text { CHE } \\
\text { NA }\end{array}$ & $\begin{array}{l}\text { Japan } \\
\text { NA }\end{array}$ & $\begin{array}{l}\text { Australia } \\
\text { NA }\end{array}$ & 1.7 \\
\hline $\begin{array}{l}\text { Low birth weight, } \% \text { of total } \\
\text { live births }\end{array}$ & $\begin{array}{l}\text { Japan } \\
9.5\end{array}$ & $\begin{array}{l}\text { US } \\
8.1\end{array}$ & $\begin{array}{l}\text { UK } \\
6.9\end{array}$ & $\begin{array}{l}\text { Germany } \\
6.6\end{array}$ & $\begin{array}{l}\text { NLD } \\
6.5\end{array}$ & $\begin{array}{l}\text { Australia } \\
6.4\end{array}$ & $\begin{array}{l}\text { Canada } \\
6.3\end{array}$ & $\begin{array}{l}\text { France } \\
6.2\end{array}$ & $\begin{array}{l}\text { Denmark } \\
5\end{array}$ & $\begin{array}{l}\text { Sweden } \\
4.4\end{array}$ & $\begin{array}{l}\text { CHE } \\
\text { NA }\end{array}$ & 6.6 \\
\hline
\end{tabular}

NA indicates not applicable. CHE indicates Switzerland; NLD, the Netherlands. See eTable 2 in Supplement 2 for data ordered by country.

a Patient self-reported data.

except for imaging. The United States performed the second highest number of MRI scans and the highest number of CT scans (118 MRIs per 1000 population compared with a mean in all 11 countries of 82 per 1000 population; 245 CTs per 1000 population compared with a mean of 151 per 1000 population).

Annual hospital discharges in the United States, at 125 per 1000 population, were just below the middle of the distribution (ranging from 84 per 1000 in Canada to 255 per 1000 in Germany, with a mean of 150 per 1000 in all 11 countries) (Figure 8). Discharges for common conditions in the United States such as acute myocardial infarction (192 per 100000 population compared with a mean of 190 per 100000 population), pneumonia (365 per 100000 population compared with a mean of 352 per 100000 population), and chronic obstructive pulmonary disease (230 per 100000 population compared with a mean of 206 per 100000 population) were similar to the means of all 11 countries. For discharges for mental and behavioral conditions, the United States was below the mean of all 11 countries (679 per 100000 population compared with a mean of 736 per 100000 population) (Figure 7). Consultation levels in the United States were below the mean at 4 visits per person per year compared with a mean of 6.6 (Figure 8).

The United States had somewhat higher levels of some common surgical procedures, such as revascularization procedures (for coronary artery bypass procedures, 79 per 100000 population compared with a mean of 54 per 100000 population), knee replacements (226 per 100000 population compared with a mean of 163 per 100000 population), cesarean deliveries (33 per 100 live births compared with a mean of 25 per 100 live births), coronary angioplasties (248 per 100000 population compared with a mean of 217 per 100000 population), and cataract surgeries (1110 per 100000 population compared with a mean of 971 per 100000 population). For a few procedures, the United States had comparable or lower rates vs the other 10 countries, such as for hip replacements (204 per 100000 population compared with a mean in all 11 countries of 207 per 100000 population).

Length of stay had less variation across countries with the exception of Japan, which had a mean all-cause length of stay of 16.9 days, far longer than in the other countries (Figure 8). The United States had relatively fewer days in the hospital compared with the mean for 3 different length-of-stay measures (all-cause hospitalization, normal neonatal delivery hospitalization, and acute myocardial infarction hospitalization) (Figure 7).

The United States had high levels of administrative burden; this was notable in particular for administrative spending, for which the United States was an outlier ( $8 \%$ of GDP spent on administration and governance compared with a mean of $3 \%$ of GDP) (eTable 1 in Supplement 1). Physicians in the United States also reported having a higher level of administrative burden than the mean of all 11 countries in 3 areas; however, this burden was high in all insurancebased systems. Fifty-four percent of surveyed physicians in the United States identified time spent on administrative issues related to insurance or claims as a major problem, 33\% reported that 
Figure 5. Workforce and Structural Capacity

\begin{tabular}{|c|c|c|c|c|c|c|c|c|c|c|c|c|}
\hline Rank (highest to lowest) & 1 & 2 & 3 & 4 & 5 & 6 & 7 & 8 & 9 & 10 & 11 & Mean \\
\hline \multicolumn{13}{|l|}{ Practicing workforce } \\
\hline $\begin{array}{l}\text { Overall physicians per } \\
1000 \text { population }\end{array}$ & $\begin{array}{l}\text { CHE } \\
4.3\end{array}$ & $\begin{array}{l}\text { Sweden } \\
4.2\end{array}$ & $\begin{array}{l}\text { Germany } \\
4.1\end{array}$ & $\begin{array}{l}\text { Denmark } \\
3.6\end{array}$ & $\begin{array}{l}\text { NLD } \\
3.5\end{array}$ & $\begin{array}{l}\text { Australia } \\
3.5\end{array}$ & $\begin{array}{l}\text { France } \\
3.1\end{array}$ & $\begin{array}{l}\text { US } \\
2.6\end{array}$ & $\begin{array}{l}\text { Canada } \\
2.6\end{array}$ & $\begin{array}{l}\text { Japan } \\
2.4\end{array}$ & $\begin{array}{l}\text { UK } \\
2.1\end{array}$ & 3.3 \\
\hline $\begin{array}{l}\text { Primary care physicians, } \\
\% \text { of total }\end{array}$ & $\begin{array}{l}\text { France } \\
54\end{array}$ & $\begin{array}{l}\text { CHE } \\
48\end{array}$ & $\begin{array}{l}\text { Canada } \\
48\end{array}$ & $\begin{array}{l}\text { NLD } \\
47\end{array}$ & $\begin{array}{l}\text { UK } \\
45\end{array}$ & $\begin{array}{l}\text { Germany } \\
45\end{array}$ & $\begin{array}{l}\text { Australia } \\
45\end{array}$ & $\begin{array}{l}\text { US } \\
43\end{array}$ & $\begin{array}{l}\text { Japan } \\
43\end{array}$ & $\begin{array}{l}\text { Sweden } \\
33\end{array}$ & $\begin{array}{l}\text { Denmark } \\
22\end{array}$ & 43 \\
\hline Specialists, \% of total & $\begin{array}{l}\text { Denmark } \\
78\end{array}$ & $\begin{array}{l}\text { Sweden } \\
67\end{array}$ & $\begin{array}{l}\text { US } \\
57\end{array}$ & $\begin{array}{l}\text { Japan } \\
57\end{array}$ & $\begin{array}{l}\text { UK } \\
55\end{array}$ & $\begin{array}{l}\text { Germany } \\
55\end{array}$ & $\begin{array}{l}\text { Australia } \\
55\end{array}$ & $\begin{array}{l}\text { NLD } \\
53\end{array}$ & $\begin{array}{l}\text { CHE } \\
52\end{array}$ & $\begin{array}{l}\text { Canada } \\
52\end{array}$ & $\begin{array}{l}\text { France } \\
46\end{array}$ & 57 \\
\hline Nurses per 1000 population & $\begin{array}{l}\text { CHE } \\
17.4\end{array}$ & $\begin{array}{l}\text { Denmark } \\
16.3\end{array}$ & $\begin{array}{l}\text { Germany } \\
13\end{array}$ & $\begin{array}{l}\text { NLD } \\
12.1\end{array}$ & $\begin{array}{l}\text { Australia } \\
11.5\end{array}$ & $\begin{array}{l}\text { Sweden } \\
11.2\end{array}$ & $\begin{array}{l}\text { US } \\
11.1\end{array}$ & $\begin{array}{l}\text { Japan } \\
10.5\end{array}$ & $\begin{array}{l}\text { Canada } \\
9.5\end{array}$ & $\begin{array}{l}\text { France } \\
9.4\end{array}$ & $\begin{array}{l}\text { UK } \\
8.2\end{array}$ & 11.8 \\
\hline \multicolumn{13}{|l|}{ Workforce remuneration, US \$ } \\
\hline Generalist physicians & $\begin{array}{l}\text { US } \\
218173\end{array}$ & $\begin{array}{l}\text { Germany } \\
154126\end{array}$ & $\begin{array}{l}\text { Canada } \\
146286\end{array}$ & $\begin{array}{l}\text { UK } \\
134671\end{array}$ & $\begin{array}{l}\text { Japan } \\
124558^{a}\end{array}$ & $\begin{array}{l}\text { France } \\
111769\end{array}$ & $\begin{array}{l}\text { NLD } \\
109586\end{array}$ & $\begin{array}{l}\text { Australia } \\
108564\end{array}$ & $\begin{array}{l}\text { Sweden } \\
86607\end{array}$ & $\begin{array}{l}\text { CHE } \\
\text { NA }\end{array}$ & $\begin{array}{l}\text { Denmark } \\
\text { NA }\end{array}$ & 133723 \\
\hline Specialist physicians & $\begin{array}{l}\text { US } \\
316000\end{array}$ & $\begin{array}{l}\text { Australia } \\
202291\end{array}$ & $\begin{array}{l}\text { NLD } \\
191995\end{array}$ & $\begin{array}{l}\text { Canada } \\
188260\end{array}$ & $\begin{array}{l}\text { Germany } \\
181243\end{array}$ & $\begin{array}{l}\text { UK } \\
171987\end{array}$ & $\begin{array}{l}\text { France } \\
153180\end{array}$ & $\begin{array}{l}\text { Denmark } \\
140505\end{array}$ & $J^{J} a a^{a}$ & $\begin{array}{l}\text { Sweden } \\
98452\end{array}$ & $\begin{array}{l}\text { CHE } \\
\text { NA }\end{array}$ & 182657 \\
\hline Nurses & $\begin{array}{l}\text { US } \\
74160\end{array}$ & $\begin{array}{l}\text { NLD } \\
65082\end{array}$ & $\begin{array}{l}\text { Australia } \\
64357\end{array}$ & $\begin{array}{l}\text { Denmark } \\
58891\end{array}$ & $\begin{array}{l}\text { Canada } \\
55349\end{array}$ & $\begin{array}{l}\text { Germany } \\
53668\end{array}$ & $\begin{array}{l}\text { UK } \\
49894\end{array}$ & $\begin{array}{l}\text { Japan } \\
44712\end{array}$ & $\begin{array}{l}\text { France } \\
42492\end{array}$ & $\begin{array}{l}\text { CHE } \\
\text { NA }\end{array}$ & $\begin{array}{l}\text { Sweden } \\
\text { NA }\end{array}$ & 51795 \\
\hline $\begin{array}{l}\text { Non-health-specific annual } \\
\text { wage, mean }\end{array}$ & $\begin{array}{l}\text { US } \\
60154\end{array}$ & $\begin{array}{l}\text { CHE } \\
60124\end{array}$ & $\begin{array}{l}\text { NLD } \\
52833\end{array}$ & $\begin{array}{l}\text { Denmark } \\
52580\end{array}$ & $\begin{array}{l}\text { Australia } \\
52063\end{array}$ & $\begin{array}{l}\text { Canada } \\
48403\end{array}$ & $\begin{array}{l}\text { Germany } \\
46389\end{array}$ & $\begin{array}{l}\text { France } \\
42992\end{array}$ & $\begin{array}{l}\text { UK } \\
42835\end{array}$ & $\begin{array}{l}\text { Sweden } \\
42816\end{array}$ & $\begin{array}{l}\text { Japan } \\
39113\end{array}$ & 49118 \\
\hline $\begin{array}{l}\text { Ratio of generalist } \\
\text { remuneration to mean wage }\end{array}$ & $\begin{array}{l}\text { US } \\
3.6\end{array}$ & $\begin{array}{l}\text { Germany } \\
3.3\end{array}$ & $\begin{array}{l}\text { UK } \\
3.1\end{array}$ & $\begin{array}{l}\text { Canada } \\
3.0\end{array}$ & $\begin{array}{l}\text { France } \\
2.6\end{array}$ & $\begin{array}{l}\text { NLD } \\
2.1\end{array}$ & $\begin{array}{l}\text { Australia } \\
2.1\end{array}$ & $\begin{array}{l}\text { Sweden } \\
2\end{array}$ & $\begin{array}{l}\text { CHE } \\
\text { NA }\end{array}$ & $\begin{array}{l}\text { Denmark } \\
\text { NA }\end{array}$ & $\begin{array}{l}\text { Japan } \\
\text { NA }\end{array}$ & 2.7 \\
\hline $\begin{array}{l}\text { Ratio of specialists } \\
\text { remuneration to mean wage }\end{array}$ & $\begin{array}{l}\text { US } \\
5.3\end{array}$ & $\begin{array}{l}\text { Germany } \\
3.9\end{array}$ & $\begin{array}{l}\text { Canada } \\
3.9\end{array}$ & $\begin{array}{l}\text { Australia } \\
3.8\end{array}$ & $\begin{array}{l}\text { France } \\
3.6\end{array}$ & $\begin{array}{l}\text { NLD } \\
3.6\end{array}$ & $\begin{array}{l}\text { UK } \\
3.4\end{array}$ & $\begin{array}{l}\text { Denmark } \\
2.6\end{array}$ & $\begin{array}{l}\text { Sweden } \\
2.3\end{array}$ & $\begin{array}{l}\text { CHE } \\
\text { NA }\end{array}$ & $\begin{array}{l}\text { Japan } \\
\mathrm{NA}\end{array}$ & 3.7 \\
\hline $\begin{array}{l}\text { Ratio of nurse remuneration } \\
\text { to mean wage }\end{array}$ & $\begin{array}{l}\text { Australia } \\
1.24\end{array}$ & $\begin{array}{l}\text { US } \\
1.23\end{array}$ & $\begin{array}{l}\text { NLD } \\
1.23\end{array}$ & $\begin{array}{l}\text { UK } \\
1.16\end{array}$ & $\begin{array}{l}\text { Germany } \\
1.16\end{array}$ & $\begin{array}{l}\text { Canada } \\
1.14\end{array}$ & $\begin{array}{l}\text { Japan } \\
1.14\end{array}$ & $\begin{array}{l}\text { Denmark } \\
1.12\end{array}$ & $\begin{array}{l}\text { France } \\
0.99\end{array}$ & $\begin{array}{l}\text { Sweden } \\
\text { NA }\end{array}$ & $\begin{array}{l}\text { CHE } \\
\text { NA }\end{array}$ & 1.1 \\
\hline \multicolumn{13}{|c|}{ Equipment per 1 million population } \\
\hline $\begin{array}{l}\text { Magnetic resonance } \\
\text { imaging units }\end{array}$ & $\begin{array}{l}\text { Japan } \\
51.7\end{array}$ & $\begin{array}{l}\text { US } \\
38.1\end{array}$ & $\begin{array}{l}\text { Germany } \\
30.5\end{array}$ & $\begin{array}{l}\text { Australia } \\
14.7 \\
\end{array}$ & $\begin{array}{l}\text { NLD } \\
12.9 \\
\end{array}$ & $\begin{array}{l}\text { France } \\
12.6 \\
\end{array}$ & $\begin{array}{l}\text { Canada } \\
8.9 \\
\end{array}$ & $\begin{array}{l}\text { UK } \\
7.2 \\
\end{array}$ & $\begin{array}{l}\text { Sweden } \\
\text { NA }\end{array}$ & $\begin{array}{l}\text { CHE } \\
\text { NA }\end{array}$ & $\begin{array}{l}\text { Denmark } \\
\text { NA }\end{array}$ & 22 \\
\hline $\begin{array}{l}\text { Computed tomography } \\
\text { units }\end{array}$ & $\begin{array}{l}\text { Japan } \\
107.2\end{array}$ & $\begin{array}{l}\text { Australia } \\
56.1\end{array}$ & $\begin{array}{l}\text { US } \\
41\end{array}$ & $\begin{array}{l}\text { Denmark } \\
37.1\end{array}$ & $\begin{array}{l}\text { CHE } \\
36.1\end{array}$ & $\begin{array}{l}\text { Germany } \\
35.3\end{array}$ & $\begin{array}{l}\text { France } \\
16.6\end{array}$ & $\begin{array}{l}\text { NLD } \\
13.3\end{array}$ & $\begin{array}{l}\text { Canada } \\
12.7\end{array}$ & $\begin{array}{l}\text { UK } \\
9.5\end{array}$ & $\begin{array}{l}\text { Sweden } \\
\text { NA }\end{array}$ & 36.5 \\
\hline $\begin{array}{l}\text { Mammography machine } \\
\text { units }\end{array}$ & $\begin{array}{l}\text { US } \\
43.3\end{array}$ & $\begin{array}{l}\text { Japan } \\
33\end{array}$ & $\begin{array}{l}\text { CHE } \\
28.3\end{array}$ & $\begin{array}{l}\text { Australia } \\
23\end{array}$ & $\begin{array}{l}\text { UK } \\
21\end{array}$ & $\begin{array}{l}\text { Canada } \\
17.3\end{array}$ & $\begin{array}{l}\text { Denmark } \\
14.2\end{array}$ & $\begin{array}{l}\text { France } \\
7.5\end{array}$ & $\begin{array}{l}\text { Germany } \\
\mathrm{NA}\end{array}$ & $\begin{array}{l}\text { Sweden } \\
\text { NA }\end{array}$ & $\begin{array}{l}\text { NLD } \\
\text { NA }\end{array}$ & 23.5 \\
\hline \multicolumn{13}{|l|}{ Beds } \\
\hline $\begin{array}{l}\text { Hospital beds per } 1000 \\
\text { population }\end{array}$ & $\begin{array}{l}\text { Japan } \\
13.2\end{array}$ & $\begin{array}{l}\text { Germany } \\
8.2\end{array}$ & $\begin{array}{l}\text { France } \\
6.1\end{array}$ & $\begin{array}{l}\text { CHE } \\
4.6\end{array}$ & $\begin{array}{l}\text { Australia } \\
3.8\end{array}$ & $\begin{array}{l}\text { NLD } \\
3.3\end{array}$ & $\begin{array}{l}\text { US } \\
2.8\end{array}$ & $\begin{array}{l}\text { UK } \\
2.7\end{array}$ & $\begin{array}{l}\text { Denmark } \\
2.7\end{array}$ & $\begin{array}{l}\text { Canada } \\
2.7\end{array}$ & $\begin{array}{l}\text { Sweden } \\
2.5\end{array}$ & 4.8 \\
\hline $\begin{array}{l}\text { Long-term beds per } 1000 \\
\text { population aged } \geq 65 \mathrm{y}\end{array}$ & $\begin{array}{l}\text { Sweden } \\
70.6\end{array}$ & $\begin{array}{l}\text { CHE } \\
67.6\end{array}$ & $\begin{array}{l}\text { NLD } \\
65.5\end{array}$ & $\begin{array}{l}\text { France } \\
59\end{array}$ & $\begin{array}{l}\text { Australia } \\
54\end{array}$ & $\begin{array}{l}\text { Canada } \\
53.7\end{array}$ & $\begin{array}{l}\text { Germany } \\
53.1\end{array}$ & $\begin{array}{l}\text { UK } \\
49.5\end{array}$ & $\begin{array}{l}\text { Denmark } \\
48.9\end{array}$ & $\begin{array}{l}\text { US } \\
38.8\end{array}$ & $\begin{array}{l}\text { Japan } \\
35.1\end{array}$ & 54.2 \\
\hline
\end{tabular}

NA indicates not applicable. CHE indicates Switzerland; NLD, the Netherlands. See eTable 3 in Supplement 2 for data ordered by country. Generalist physicians are defined as any practicing physician registered in his or her country as a generalist physician or a specialist in the field of family medicine, pediatrics, geriatrics, or internal medicine and excludes students, interns, and nonpracticing physicians. Remuneration numbers may be an underestimate in some countries (eg, Canada) because they do not account for practice expenses for self-employed physicians. Japan is excluded from remuneration means with the exception of nursing. Definitions of specialist and generalist physicians in regard to remuneration were taken from the Organisation for Economic Co-operation and Development.

a The number for Japan, 124558, is a combined total of generalists and specialists.

b In 2016 constant prices at 2016 US dollar purchasing power parities. "time spent on administrative issues related to reporting clinical or quality data to government or other agencies is a major problem," and $16 \%$ reported having spent "a lot of time on paperwork or disputes related to medical bills."

\section{Pharmaceuticals}

Among the 11 countries, the United States had the highest pharmaceutical spending per capita at $\$ 1443$, with Switzerland following at $\$ 939$ and a mean of $\$ 749$ for all 11 countries (Figure 9 and eTable 5 in Supplement 2). Retail spending per capita was also highest in the United States at \$1026, representing about 71\% of the total, which was consistent with the group mean at 72\% (Figure 9). For 4 pharmaceuticals (Crestor, Lantus, Advair, and Humira) used for common conditions, the United States had higher prices than all other countries; for 3 of these, the US price was more than double the next highest price. With respect to a measure of innovation, the United States and Switzerland had the highest number of new chemical entities at 111 and 26, respectively. The United States accounted for $57 \%$ of total global production of new chemical entities. No estimates were available for Canada, Australia, Sweden, the Netherlands, and Denmark. The United States also had high generic penetration at $84 \%$ of the total pharmaceutical market, which was comparable with markets in the United Kingdom and Germany. Australia and the Netherlands had low generic penetration at $30 \%$ and $17 \%$, respectively. Despite having the highest rate of generic penetration, the amount that the United States spent on generic products as a percentage of total pharmaceutical spending was similar to other countries, suggesting that brand-name 


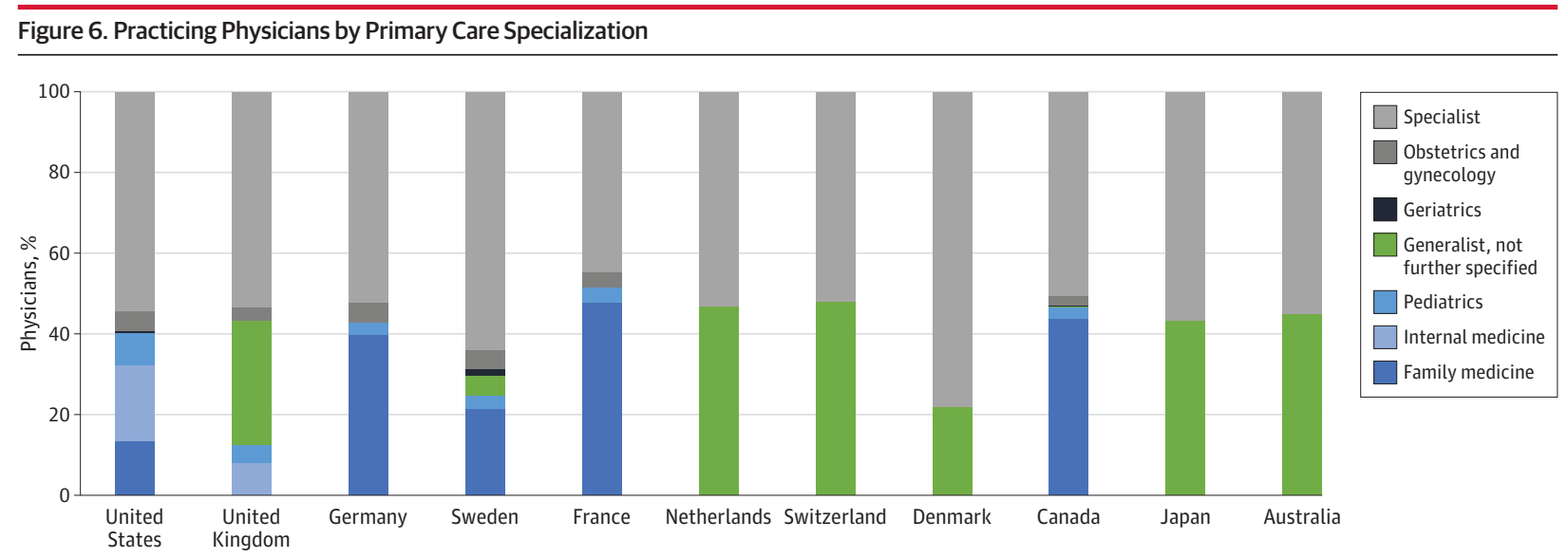

The total number of physicians, or 100\%, differs for each country. Data are 2017 or closest available year. A functionality-based definition to identify primary care was used to identify physicians as those who provide a set of activities whose functions define the boundaries of primary care. This included chronic, preventive, and acute care in both inpatient and outpatient settings irrespective of disease: disease prevention, early detection and diagnosis, treatment and management, care coordination and integration, and health maintenance, counseling, and/or patient education. The clinicians were often a patient's first point of contact for the health system with some degree of longitudinal responsibility for the patient. This definition was used to reach out to country experts and identify who is considered a primary care clinician in each respective country. National workforce data or Eurostat data, where available, was then categorized according to expert responses. Physicians specializing in obstetrics and gynecology are categorized as specialists for all countries but have their own category in this figure. pharmaceuticals were largely responsible for high overall spending. These countries have notably different ways in how they finance pharmaceuticals; there is considerable variation in both the share covered by private insurance and the percentage covered by private out-of-pocket spending. The United States had high levels of private spending (36\% compared with a mean of $8 \%$ ), although similar to Canada (30\%), and was below the mean for out-ofpocket spending at 30\% (compared with a mean of 36\%). With regard to antibiotic prescriptions, which are often considered a measure of inappropriate treatment, the United States was above the mean, with a defined daily dose (average maintenance dose per day for a drug used for its main indication in adults) of 24 compared with a mean of 20.2 .

\section{Access and Quality}

Relative to comparison countries, US performance varied on quality and access measures (Figure 10 and eTable 6 in Supplement 2). Of the other countries examined, the United States was the only one in which a sizeable minority (approximately $10 \%$ ) of individuals lacked coverage for basic health care services (Figure 1). For the 3 access measures (ability to get same- or next-day care when sick, 2-month wait time to see a specialist, and adequate time spent with regular physician) the United States generally performed better than the other countries. In the United States, $51 \%$ were able to get sameor next-day care compared with a mean of $57 \%$ in all 11 countries. In the United States, $6 \%$ had a wait time of 2 months or more to see a specialist compared with mean of $13 \%$ in all 11 countries. Waiting times to see a specialist were longer for national health service and single-payer systems (ie, percentage with wait times longer than 2 months: Canada, 39\%; United Kingdom, 19\%; Sweden, 19\%) compared with insurance-based systems (Netherlands, 7\%; Switzerland, 9\%; Germany, 3\%; France, 4\%), with a mean of $13 \%$. The United States ranked near the mean for patients reporting hav- ing spent adequate time with their regular physician (81\% compared with a mean of $83 \%$ in all 11 countries).

The United States had relatively high screening rates for breast cancer ( $81 \%$ compared with a mean of $67 \%$ in all 11 countries) but lower rates of measles immunization ( $92 \%$ compared with a mean of 94\%) (Figure 10). The United States had considerably lower rates of all 4 clinical outcome measures than the other countries. Thirtyday mortality for ischemic stroke was 4.2 per 100 patients in the United States compared with a mean of 7.9 per 100 patients in all 11 countries. For obstetric trauma without instrument, the United States had 1.5 cases per 100 deliveries, whereas the mean was 2.3 per 100 deliveries in all 11 countries. The United States had high avoidable hospitalizations for diabetes and asthma relative to comparison countries (191.0 per 100000 population compared with a mean of 125.6 per 100000 population for diabetes, and 89.7 per 100000 population compared with a mean of 42.4 per 100000 population for asthma). When accounting for disease prevalence, the rate of US hospitalizations for diabetes was similar to that of other countries, although hospitalizations for asthma were highest in the United States, closely followed by the United Kingdom. Relative to the other countries, the US public reported the lowest satisfaction with their health system, with only $19 \%$ reporting that the system works well.

\section{US Disaggregated Data}

When access and quality measures were disaggregated by payer, the United States performed slightly worse for those covered by Medicaid, but performance was uneven across insurance groups (eTable 4 in Supplement 1). Nine percent of both privately insured individuals and those insured by Medicaid reported a 2-month wait time to see a specialist; this was lower than rates for Medicare patients (11\%) but higher than for uninsured individuals ( $8 \%)$. On selected prevention measures, such as measles immunization, privately insured individuals had the highest rate of coverage at $95 \%$, 


\begin{tabular}{|c|c|c|c|c|c|c|c|c|c|c|c|c|}
\hline \multicolumn{13}{|l|}{ Figure 7. Utilization } \\
\hline Rank (highest to lowest) & 1 & 2 & 3 & 4 & 5 & 6 & 7 & 8 & 9 & 10 & 11 & Mean \\
\hline \multicolumn{13}{|c|}{ Discharges per 100000 population } \\
\hline Acute myocardial infarction & $\begin{array}{l}\text { Germany } \\
287\end{array}$ & $\begin{array}{l}\text { Sweden } \\
273\end{array}$ & $\begin{array}{l}\text { CHE } \\
223\end{array}$ & $\begin{array}{l}\text { Australia } \\
196\end{array}$ & $\begin{array}{l}\text { Canada } \\
193\end{array}$ & $\begin{array}{l}\text { US } \\
192\end{array}$ & $\begin{array}{l}\text { NLD } \\
175\end{array}$ & $\begin{array}{l}\text { Denmark } \\
174\end{array}$ & $\begin{array}{l}\text { UK } \\
160\end{array}$ & $\begin{array}{l}\text { France } \\
124\end{array}$ & $\begin{array}{l}\text { Japan } \\
89\end{array}$ & 190 \\
\hline Mental and behavioral & $\begin{array}{l}\text { Germany } \\
1719\end{array}$ & $\begin{array}{l}\text { CHE } \\
1182\end{array}$ & $\begin{array}{l}\text { Sweden } \\
1068\end{array}$ & $\begin{array}{l}\text { Denmark } \\
892\end{array}$ & $\begin{array}{l}\text { Australia } \\
856\end{array}$ & $\begin{array}{l}\text { US } \\
679\end{array}$ & $\begin{array}{l}\text { Canada } \\
629\end{array}$ & $\begin{array}{l}\text { France } \\
368\end{array}$ & $\begin{array}{l}\text { Japan } \\
319\end{array}$ & $\begin{array}{l}\text { UK } \\
269\end{array}$ & $\begin{array}{l}\text { NLD } \\
119\end{array}$ & 736 \\
\hline Pneumonia & $\begin{array}{l}\text { Denmark } \\
567\end{array}$ & $\begin{array}{l}\text { UK } \\
459\end{array}$ & $\begin{array}{l}\text { Sweden } \\
432\end{array}$ & $\begin{array}{l}\text { Germany } \\
380\end{array}$ & $\begin{array}{l}\text { Japan } \\
378\end{array}$ & $\begin{array}{l}\text { US } \\
365\end{array}$ & $\begin{array}{l}\text { Australia } \\
338\end{array}$ & $\begin{array}{l}\text { France } \\
271\end{array}$ & $\begin{array}{l}\text { CHE } \\
269\end{array}$ & $\begin{array}{l}\text { NLD } \\
224\end{array}$ & $\begin{array}{l}\text { Canada } \\
187\end{array}$ & 352 \\
\hline $\begin{array}{l}\text { Chronic obstructive } \\
\text { pulmonary disease }\end{array}$ & $\begin{array}{l}\text { Germany } \\
352\end{array}$ & $\begin{array}{l}\text { Australia } \\
286\end{array}$ & $\begin{array}{l}\text { UK } \\
251\end{array}$ & $\begin{array}{l}\text { Canada } \\
241\end{array}$ & $\begin{array}{l}\text { Denmark } \\
234\end{array}$ & $\begin{array}{l}\text { US } \\
230\end{array}$ & $\begin{array}{l}\text { Sweden } \\
186\end{array}$ & $\begin{array}{l}\text { NLD } \\
161\end{array}$ & $\begin{array}{l}\text { CHE } \\
142\end{array}$ & $\begin{array}{l}\text { France } \\
138\end{array}$ & $\begin{array}{l}\text { Japan } \\
45\end{array}$ & 206 \\
\hline \multicolumn{13}{|c|}{ Examinations per 1000 population } \\
\hline Magnetic resonance imaging & $\begin{array}{l}\text { Germany } \\
131\end{array}$ & $\begin{array}{l}\text { US } \\
118\end{array}$ & $\begin{array}{l}\text { Japan } \\
112\end{array}$ & $\begin{array}{l}\text { France } \\
105\end{array}$ & $\begin{array}{l}\text { Denmark } \\
82\end{array}$ & $\begin{array}{l}\text { CHE } \\
70\end{array}$ & $\begin{array}{l}\text { Canada } \\
56\end{array}$ & $\begin{array}{l}\text { UK } \\
53\end{array}$ & $\begin{array}{l}\text { NLD } \\
52\end{array}$ & $\begin{array}{l}\text { Australia } \\
41\end{array}$ & $\begin{array}{l}\text { Sweden } \\
\text { NA }\end{array}$ & 82 \\
\hline Computed tomography & $\begin{array}{l}\text { US } \\
245\end{array}$ & $\begin{array}{l}\text { Japan } \\
231\end{array}$ & $\begin{array}{l}\text { France } \\
197\end{array}$ & $\begin{array}{l}\text { Denmark } \\
162\end{array}$ & $\begin{array}{l}\text { Canada } \\
153\end{array}$ & $\begin{array}{l}\text { Germany } \\
144\end{array}$ & $\begin{array}{l}\text { Australia } \\
120\end{array}$ & $\begin{array}{l}\text { CHE } \\
100\end{array}$ & $\begin{array}{l}\text { NLD } \\
81\end{array}$ & $\begin{array}{l}\text { UK } \\
79\end{array}$ & $\begin{array}{l}\text { Sweden } \\
\text { NA }\end{array}$ & 151 \\
\hline \multicolumn{13}{|l|}{ Surgical procedures } \\
\hline $\begin{array}{l}\text { Total hip replacement } \\
\text { per } 100000 \text { population }\end{array}$ & $\begin{array}{l}\text { CHE } \\
292\end{array}$ & $\begin{array}{l}\text { Germany } \\
283\end{array}$ & $\begin{array}{l}\text { Denmark } \\
237\end{array}$ & $\begin{array}{l}\text { France } \\
236\end{array}$ & $\begin{array}{l}\text { Sweden } \\
234\end{array}$ & $\begin{array}{l}\text { NLD } \\
216\end{array}$ & $\begin{array}{l}\text { US } \\
204\end{array}$ & $\begin{array}{l}\text { UK } \\
183\end{array}$ & $\begin{array}{l}\text { Australia } \\
171\end{array}$ & $\begin{array}{l}\text { Canada } \\
136\end{array}$ & $\begin{array}{l}\text { Japan } \\
90\end{array}$ & 207 \\
\hline $\begin{array}{l}\text { Total knee replacement } \\
\text { per } 100000 \text { population }\end{array}$ & $\begin{array}{l}\text { US } \\
226\end{array}$ & $\begin{array}{l}\text { Germany } \\
190\end{array}$ & $\begin{array}{l}\text { Australia } \\
180\end{array}$ & $\begin{array}{l}\text { CHE } \\
176\end{array}$ & $\begin{array}{l}\text { Denmark } \\
168\end{array}$ & $\begin{array}{l}\text { Canada } \\
166\end{array}$ & $\begin{array}{l}\text { France } \\
145\end{array}$ & $\begin{array}{l}\text { UK } \\
141\end{array}$ & $\begin{array}{l}\text { Sweden } \\
124\end{array}$ & $\begin{array}{l}\text { NLD } \\
118\end{array}$ & $\begin{array}{l}\text { Japan } \\
\text { NA }\end{array}$ & 163 \\
\hline $\begin{array}{l}\text { Hysterectomy per } 100000 \\
\text { women }\end{array}$ & $\begin{array}{l}\text { Germany } \\
301\end{array}$ & $\begin{array}{l}\text { CHE } \\
291\end{array}$ & $\begin{array}{l}\text { US } \\
266\end{array}$ & $\begin{array}{l}\text { Australia } \\
262\end{array}$ & $\begin{array}{l}\text { Canada } \\
232\end{array}$ & $\begin{array}{l}\text { Denmark } \\
197\end{array}$ & $\begin{array}{l}\text { Sweden } \\
186\end{array}$ & $\begin{array}{l}\text { France } \\
182\end{array}$ & $\begin{array}{l}\text { NLD } \\
167\end{array}$ & $\begin{array}{l}\text { UK } \\
161\end{array}$ & $\begin{array}{l}\text { Japan } \\
\text { NA }\end{array}$ & 225 \\
\hline $\begin{array}{l}\text { Cesarean delivery per } \\
100 \text { live births }\end{array}$ & $\begin{array}{l}\text { US } \\
33\end{array}$ & $\begin{array}{l}\text { CHE } \\
33\end{array}$ & $\begin{array}{l}\text { Australia } \\
32\end{array}$ & $\begin{array}{l}\text { Germany } \\
31\end{array}$ & $\begin{array}{l}\text { Canada } \\
26\end{array}$ & $\begin{array}{l}\text { UK } \\
23\end{array}$ & $\begin{array}{l}\text { France } \\
21\end{array}$ & $\begin{array}{l}\text { Denmark } \\
21\end{array}$ & $\begin{array}{l}\text { Japan } \\
18\end{array}$ & $\begin{array}{l}\text { Sweden } \\
17\end{array}$ & $\begin{array}{l}\text { NLD } \\
16\end{array}$ & 25 \\
\hline $\begin{array}{l}\text { Cataract surgery per } 100000 \\
\text { population }\end{array}$ & $\begin{array}{l}\text { France } \\
1207\end{array}$ & $\begin{array}{l}\text { US } \\
1110\end{array}$ & $\begin{array}{l}\text { Canada } \\
1060\end{array}$ & $\begin{array}{l}\text { Australia } \\
1060\end{array}$ & $\begin{array}{l}\text { Denmark } \\
1037\end{array}$ & $\begin{array}{l}\text { Sweden } \\
1029\end{array}$ & $\begin{array}{l}\text { Germany } \\
1027\end{array}$ & $\begin{array}{l}\text { NLD } \\
1005\end{array}$ & $\begin{array}{l}\text { UK } \\
736\end{array}$ & $\begin{array}{l}\text { CHE } \\
438\end{array}$ & $\begin{array}{l}\text { Japan } \\
\text { NA }\end{array}$ & 971 \\
\hline \multicolumn{13}{|c|}{ Cardiovascular procedures per 100000 population } \\
\hline $\begin{array}{l}\text { Coronary artery bypass graft } \\
\text { surgery }\end{array}$ & $\begin{array}{l}\text { US } \\
79\end{array}$ & $\begin{array}{l}\text { Denmark } \\
73\end{array}$ & $\begin{array}{l}\text { NLD } \\
69\end{array}$ & $\begin{array}{l}\text { Germany } \\
64\end{array}$ & $\begin{array}{l}\text { Canada } \\
58\end{array}$ & $\begin{array}{l}\text { Australia } \\
54\end{array}$ & $\begin{array}{l}\text { Sweden } \\
31\end{array}$ & $\begin{array}{l}\text { France } \\
29\end{array}$ & $\begin{array}{l}\text { UK } \\
26\end{array}$ & $\begin{array}{l}\text { CHE } \\
\text { NA }\end{array}$ & $\begin{array}{l}\text { Japan } \\
\text { NA }\end{array}$ & 54 \\
\hline Coronary angioplasty & $\begin{array}{l}\text { France } \\
393\end{array}$ & $\begin{array}{l}\text { US } \\
248\end{array}$ & $\begin{array}{l}\text { NLD } \\
248\end{array}$ & $\begin{array}{l}\text { France } \\
237\end{array}$ & $\begin{array}{l}\text { Sweden } \\
205\end{array}$ & $\begin{array}{l}\text { Japan } \\
193\end{array}$ & $\begin{array}{l}\text { Denmark } \\
190\end{array}$ & $\begin{array}{l}\text { Australia } \\
172\end{array}$ & $\begin{array}{l}\text { Canada } \\
157\end{array}$ & $\begin{array}{l}\text { UK } \\
128\end{array}$ & $\begin{array}{l}\text { CHE } \\
\text { NA }\end{array}$ & 217 \\
\hline \multicolumn{13}{|l|}{ Length of stay per capita, mean, d } \\
\hline Normal delivery & $\begin{array}{l}\text { Japan } \\
5.7\end{array}$ & $\begin{array}{l}\text { France } \\
4.1\end{array}$ & $\begin{array}{l}\text { CHE } \\
3.6\end{array}$ & $\begin{array}{l}\text { Germany } \\
2.9\end{array}$ & $\begin{array}{l}\text { Denmark } \\
2.7\end{array}$ & $\begin{array}{l}\text { Australia } \\
2.7\end{array}$ & $\begin{array}{l}\text { Sweden } \\
2.3\end{array}$ & $\begin{array}{l}\text { US } \\
2\end{array}$ & $\begin{array}{l}\text { NLD } \\
1.9\end{array}$ & $\begin{array}{l}\text { Canada } \\
1.6\end{array}$ & $\begin{array}{l}\text { UK } \\
1.5\end{array}$ & 2.8 \\
\hline Acute myocardial infarction & $\begin{array}{l}\text { Germany } \\
10.3\end{array}$ & $\begin{array}{l}\text { CHE } \\
7.3\end{array}$ & $\begin{array}{l}\text { UK } \\
7.1\end{array}$ & $\begin{array}{l}\text { France } \\
6\end{array}$ & $\begin{array}{l}\text { NLD } \\
5.6\end{array}$ & $\begin{array}{l}\text { Canada } \\
5.5\end{array}$ & $\begin{array}{l}\text { US } \\
5.4\end{array}$ & $\begin{array}{l}\text { Australia } \\
5.4\end{array}$ & $\begin{array}{l}\text { Sweden } \\
4.7\end{array}$ & $\begin{array}{l}\text { Denmark } \\
3.9\end{array}$ & $\begin{array}{l}\text { Japan } \\
\text { NA }\end{array}$ & 6.1 \\
\hline
\end{tabular}

NA indicates not applicable. CHE indicates Switzerland; NLD, the Netherlands. See eTable 4 in Supplement 2 for data ordered by country.

whereas for breast screening, Medicare beneficiaries had the highest rates (82\%). Conversely, Medicare beneficiaries had the highest rates of mortality for both ischemic stroke and acute myocardial infarction. There was also variability in avoidable admissions: patients with Medicaid insurance had the highest number of asthma-related admissions and Medicare beneficiaries had the highest number of admissions for diabetes. When disaggregated by income or race, life expectancy was significantly different among US groups, with nonwhite and poorer populations having shorter life expectancies (eTables 3 and 4 in Supplement 1).

\section{Equity}

The United States had the highest horizontal inequity, indicating the most inequitable access to physicians when adjusted for need. The United States had an $11 \%$ rate of out-of-pocket spending as a percentage of total national health spending (compared with a mean of $13 \%$ in all 11 countries) and a $2.6 \%$ rate as a percentage of household consumption (compared with a mean of 2.4\%) (Figure 11 and eTable 7 in Supplement 2). However, the United States had a higher proportion of unmet need in the population, with $22.3 \%$ of the popu- lation reporting that they missed a consultation because of cost compared with the mean of $9.4 \%$ for all 11 study countries. Given that the system has free access at the point of entry, the United Kingdom reported one of the lowest levels of barriers to accessing health services, but the level was higher than in both Germany and Sweden.

\section{Discussion}

In this study based on data primarily from 2013-2016, the United States spent approximately twice as much as other high-income countries on medical care and fared worse on common population health outcomes such as life expectancy and infant mortality. However, the main findings of this comparison were that, contrary to some explanations for high spending, US social spending and health care utilization were relatively similar to other high-income nations. Although utilization of some surgical procedures (such as coronary angioplasty, total knee replacement, and cesarean delivery) was higher in the United States, this utilization did not appear to explain a large part of the higher spending in the United States. 
Figure 8. Performance on Key Measures of Utilization

A Hospital discharges

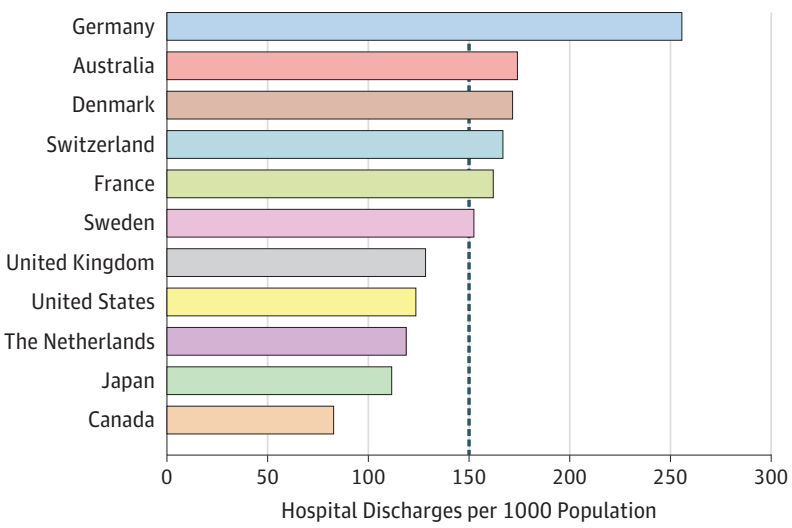

C Hospital bed days

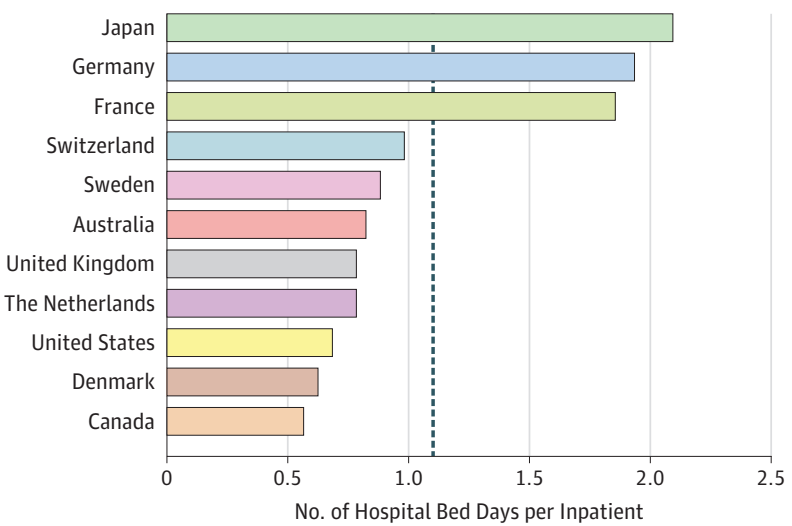

B Consultations ${ }^{\mathrm{a}}$

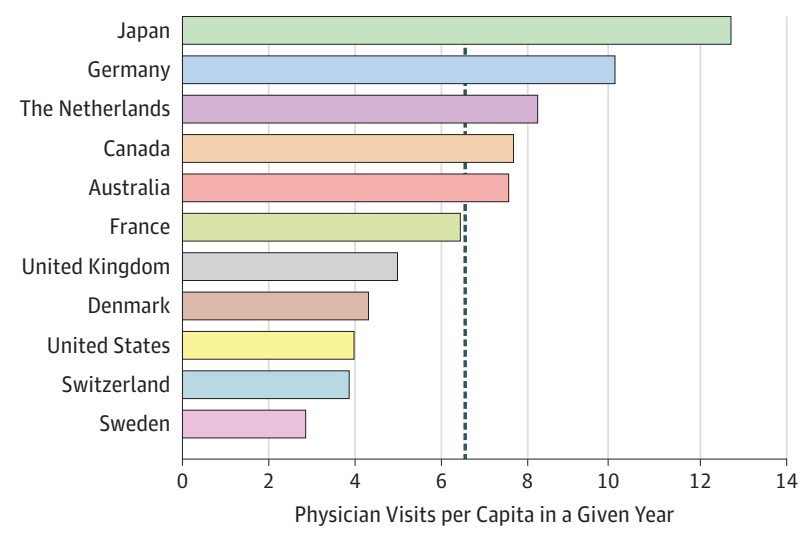

D All-cause length of stay

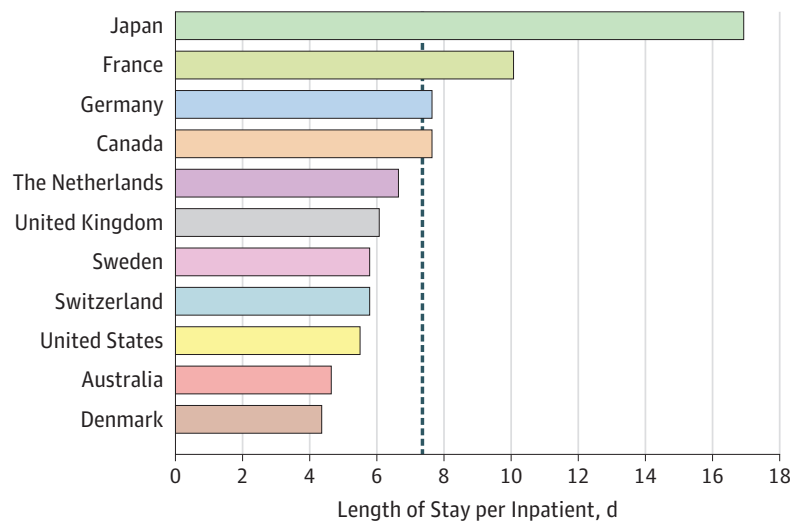

The vertical dashed lines indicate mean values.

${ }^{a}$ Consultations is the mean number of consultations or visits with a physician per person per year in all care delivery settings.

The data also suggest that some of the more common explanations about higher health care spending in the United States, such as underinvestment in social programs, the low primary care/specialist mix, the fee-for-service system encouraging high volumes of care, or defensive medicine leading to overutilization, did not appear to be major drivers of the substantially higher US health care spending compared with other high-income countries. Instead, the data suggest that the main driving factors were likely related to prices, including prices of physician and hospital services, pharmaceuticals, and diagnostic tests, which likely also affected access to care. In addition, administrative costs appeared much higher in the United States. These findings indicate that efforts targeting utilization alone are unlikely to reduce the gap in spending between the United States and other high-income countries, and a more concerted effort to reduce prices and administrative costs is likely needed.

Several findings in this report may be surprising to policy makers. There is broad consensus among US policy makers that the United States spends too much on health services and too little on social services. This analysis showed that US social spending appears to be similar to that in other high-income OECD countries. This finding calls into question the belief that higher health care spending is due to a lack of investment in social determinants. In particular, given that the United States did not appear to be an outlier with regard to utilization of services, it is unlikely that a lack of social spending results in higher health care spending due to a misallocation of resources that results in greater need (and overutilization).

Another common perception among policy makers is that the US system is often perceived to be disproportionately driven by specialist care. However, the number of specialist practitioners in the US system, both as an absolute number and a percentage, was not considerably different from comparison countries. One explanation may be differences in the way primary care services are delivered in other countries. In many other countries, nurses and allied health professionals may make up a higher proportion of the health care workforce, ${ }^{21}$ although we did not find substantially higher numbers of nurses in the other countries. The extent to which this explains variation in numbers of physicians across systems is unclear, but it is unlikely to fully account for why the United States is not an outlier.

Although the ratio of primary care physicians to specialists was similar between the United States and other high-income countries, 
Figure 9. Pharmaceuticals

\begin{tabular}{|c|c|c|c|c|c|c|c|c|c|c|c|c|}
\hline Rank (highest to lowest) & 1 & 2 & 3 & 4 & 5 & 6 & 7 & 8 & 9 & 10 & 11 & Mean \\
\hline Total spending per capita, US $\$$ & $\begin{array}{l}\text { US } \\
1443\end{array}$ & $\begin{array}{l}\text { CHE } \\
939\end{array}$ & $\begin{array}{l}\text { Japan } \\
837\end{array}$ & $\begin{array}{l}\text { UK } \\
779\end{array}$ & $\begin{array}{l}\text { France } \\
697\end{array}$ & $\begin{array}{l}\text { Denmark } \\
675\end{array}$ & $\begin{array}{l}\text { Germany } \\
667\end{array}$ & $\begin{array}{l}\text { Canada } \\
613\end{array}$ & $\begin{array}{l}\text { Sweden } \\
566\end{array}$ & $\begin{array}{l}\text { Australia } \\
560\end{array}$ & $\begin{array}{l}\text { NLD } \\
466\end{array}$ & 749 \\
\hline $\begin{array}{l}\text { Retail pharmaceutical spending } \\
\text { per capita, US } \$\end{array}$ & $\begin{array}{l}\text { US } \\
1026\end{array}$ & $\begin{array}{l}\text { CHE } \\
776\end{array}$ & $\begin{array}{l}\text { Canada } \\
587\end{array}$ & $\begin{array}{l}\text { Denmark } \\
573\end{array}$ & $\begin{array}{l}\text { France } \\
541\end{array}$ & $\begin{array}{l}\text { Sweden } \\
501\end{array}$ & $\begin{array}{l}\text { Germany } \\
480\end{array}$ & $\begin{array}{l}\text { Japan } \\
443\end{array}$ & $\begin{array}{l}\text { UK } \\
383\end{array}$ & $\begin{array}{l}\text { Australia } \\
346\end{array}$ & $\begin{array}{l}\text { NLD } \\
292\end{array}$ & 541 \\
\hline \multicolumn{13}{|l|}{ Prices, US $\$$ per $\mathrm{mo}^{\mathrm{a}}$} \\
\hline Crestor (cholesterol) & $\begin{array}{l}\text { US } \\
86\end{array}$ & $\begin{array}{l}\text { Germany } \\
41\end{array}$ & $\begin{array}{l}\text { Canada } \\
32\end{array}$ & $\begin{array}{l}\text { Japan } \\
29\end{array}$ & $\begin{array}{l}\text { UK } \\
26\end{array}$ & $\begin{array}{l}\text { France } \\
20\end{array}$ & $\begin{array}{l}\text { Australia } \\
9\end{array}$ & $\begin{array}{l}\text { Sweden } \\
\text { NA }\end{array}$ & $\begin{array}{l}\text { NLD } \\
\text { NA }\end{array}$ & $\begin{array}{l}\text { CHE } \\
\text { NA }\end{array}$ & $\begin{array}{l}\text { Denmark } \\
\text { NA }\end{array}$ & 35 \\
\hline Lantus (diabetes) & $\begin{array}{l}\text { US } \\
186\end{array}$ & $\begin{array}{l}\text { Canada } \\
67\end{array}$ & $\begin{array}{l}\text { UK } \\
64\end{array}$ & $\begin{array}{l}\text { Japan } \\
64\end{array}$ & $\begin{array}{l}\text { Germany } \\
61\end{array}$ & $\begin{array}{l}\text { Australia } \\
54\end{array}$ & $\begin{array}{l}\text { France } \\
47\end{array}$ & $\begin{array}{l}\text { Sweden } \\
\text { NA }\end{array}$ & $\begin{array}{l}\text { NLD } \\
\text { NA }\end{array}$ & $\begin{array}{l}\text { CHE } \\
\text { NA }\end{array}$ & $\begin{array}{l}\text { Denmark } \\
\text { NA }\end{array}$ & 78 \\
\hline Advair (asthma) & $\begin{array}{l}\text { US } \\
155\end{array}$ & $\begin{array}{l}\text { Canada } \\
74\end{array}$ & $\begin{array}{l}\text { Japan } \\
51\end{array}$ & $\begin{array}{l}\text { Germany } \\
38\end{array}$ & $\begin{array}{l}\text { France } \\
35\end{array}$ & $\begin{array}{l}\text { Australia } \\
29\end{array}$ & $\begin{array}{l}\text { UK } \\
\text { NA }\end{array}$ & $\begin{array}{l}\text { Sweden } \\
\text { NA }\end{array}$ & $\begin{array}{l}\text { NLD } \\
\text { NA }\end{array}$ & $\begin{array}{l}\text { CHE } \\
\text { NA }\end{array}$ & $\begin{array}{l}\text { Denmark } \\
\text { NA }\end{array}$ & 64 \\
\hline Humira (rheumatoid arthritis) & $\begin{array}{l}\text { US } \\
2505\end{array}$ & $\begin{array}{l}\text { Germany } \\
1749\end{array}$ & $\begin{array}{l}\text { Australia } \\
1243\end{array}$ & $\begin{array}{l}\text { Canada } \\
1164\end{array}$ & $\begin{array}{l}\text { UK } \\
1158\end{array}$ & $\begin{array}{l}\text { France } \\
982\end{array}$ & $\begin{array}{l}\text { Japan } \\
980\end{array}$ & $\begin{array}{l}\text { Sweden } \\
\text { NA }\end{array}$ & $\begin{array}{l}\text { NLD } \\
\text { NA }\end{array}$ & $\begin{array}{l}\text { CHE } \\
\text { NA }\end{array}$ & $\begin{array}{l}\text { Denmark } \\
\text { NA }\end{array}$ & 1436 \\
\hline New chemical entities, No. ${ }^{\mathrm{b}}$ & $\begin{array}{l}\text { US } \\
111\end{array}$ & $\begin{array}{l}\text { CHE } \\
26\end{array}$ & $\begin{array}{l}\text { Japan } \\
18\end{array}$ & $\begin{array}{l}\text { UK } \\
16\end{array}$ & $\begin{array}{l}\text { Germany } \\
12\end{array}$ & $\begin{array}{l}\text { France } \\
11\end{array}$ & $\begin{array}{l}\text { Sweden } \\
\text { NA }\end{array}$ & $\begin{array}{l}\text { NLD } \\
\text { NA }\end{array}$ & $\begin{array}{l}\text { Denmark } \\
\text { NA }\end{array}$ & $\begin{array}{l}\text { Canada } \\
\text { NA }\end{array}$ & $\begin{array}{l}\text { Australia } \\
\text { NA }\end{array}$ & NA \\
\hline \multicolumn{13}{|c|}{ Pharmaceutical expenditure by financing type, \% of total spending } \\
\hline Public spending & $\begin{array}{l}\text { France } \\
80\end{array}$ & $\begin{array}{l}\text { Germany } \\
75\end{array}$ & $\begin{array}{l}\text { Japan } \\
71\end{array}$ & $\begin{array}{l}\text { UK } \\
66\end{array}$ & $\begin{array}{l}\text { NLD } \\
65\end{array}$ & $\begin{array}{l}\text { Sweden } \\
52\end{array}$ & $\begin{array}{l}\text { Australia } \\
49\end{array}$ & $\begin{array}{l}\text { CHE } \\
43\end{array}$ & $\begin{array}{l}\text { Denmark } \\
43\end{array}$ & $\begin{array}{l}\text { Canada } \\
36\end{array}$ & $\begin{array}{l}\text { US } \\
34\end{array}$ & 56 \\
\hline Private insurance & $\begin{array}{l}\text { US } \\
36\end{array}$ & $\begin{array}{l}\text { Canada } \\
30\end{array}$ & $\begin{array}{l}\text { CHE } \\
8\end{array}$ & $\begin{array}{l}\text { Denmark } \\
8\end{array}$ & $\begin{array}{l}\text { Germany } \\
7\end{array}$ & $\begin{array}{l}\text { NLD } \\
2\end{array}$ & $\begin{array}{l}\text { France } \\
1\end{array}$ & $\begin{array}{l}\text { Japan } \\
1\end{array}$ & $\begin{array}{l}\text { UK } \\
0\end{array}$ & $\begin{array}{l}\text { Sweden } \\
0\end{array}$ & $\begin{array}{l}\text { Australia } \\
0\end{array}$ & 8 \\
\hline Private out-of-pocket spending & $\begin{array}{l}\text { CHE } \\
51\end{array}$ & $\begin{array}{l}\text { Denmark } \\
51\end{array}$ & $\begin{array}{l}\text { Australia } \\
50\end{array}$ & $\begin{array}{l}\text { Sweden } \\
48\end{array}$ & $\begin{array}{l}\text { UK } \\
36\end{array}$ & $\begin{array}{l}\text { Canada } \\
34\end{array}$ & $\begin{array}{l}\text { NLD } \\
33\end{array}$ & $\begin{array}{l}\text { US } \\
30\end{array}$ & $\begin{array}{l}\text { Japan } \\
28\end{array}$ & $\begin{array}{l}\text { France } \\
19\end{array}$ & $\begin{array}{l}\text { Germany } \\
18\end{array}$ & 36 \\
\hline \multicolumn{13}{|l|}{ Share of generics, $\%$ of total ${ }^{c}$} \\
\hline Volume & $\begin{array}{l}\text { US } \\
84 \\
\end{array}$ & $\begin{array}{l}\text { UK } \\
83 \\
\end{array}$ & $\begin{array}{l}\text { Germany } \\
80\end{array}$ & $\begin{array}{l}\text { France } \\
70\end{array}$ & $\begin{array}{l}\text { Canada } \\
70\end{array}$ & $\begin{array}{l}\text { Japan } \\
56\end{array}$ & \begin{tabular}{|l} 
CHE \\
54 \\
\end{tabular} & $\begin{array}{l}\text { Denmark } \\
54\end{array}$ & $\begin{array}{l}\text { Sweden } \\
44\end{array}$ & $\begin{array}{l}\text { Australia } \\
30\end{array}$ & $\begin{array}{l}\text { NLD } \\
17\end{array}$ & 58 \\
\hline Value & $\begin{array}{l}\text { Germany } \\
37\end{array}$ & $\begin{array}{l}\text { UK } \\
33\end{array}$ & $\begin{array}{l}\text { Japan } \\
33\end{array}$ & $\begin{array}{l}\text { Canada } \\
29\end{array}$ & $\begin{array}{l}\text { US } \\
28\end{array}$ & $\begin{array}{l}\text { France } \\
16\end{array}$ & \begin{tabular}{|l} 
NLD \\
16
\end{tabular} & $\begin{array}{l}\text { Sweden } \\
15\end{array}$ & $\begin{array}{l}\text { Australia } \\
15\end{array}$ & $\begin{array}{l}\text { CHE } \\
14 \\
\end{array}$ & $\begin{array}{l}\text { Denmark } \\
14\end{array}$ & 23 \\
\hline $\begin{array}{l}\text { Antibiotic prescribing, defined } \\
\text { daily doses per } 1000 \text { population }\end{array}$ & $\begin{array}{l}\text { France } \\
29.9\end{array}$ & $\begin{array}{l}\text { Australia } \\
28.3\end{array}$ & $\begin{array}{l}\text { Canada } \\
25\end{array}$ & $\begin{array}{l}\text { US } \\
24\end{array}$ & $\begin{array}{l}\text { UK } \\
20.1\end{array}$ & $\begin{array}{l}\text { Denmark } \\
16.6\end{array}$ & $\begin{array}{l}\text { Germany } \\
14.4\end{array}$ & $\begin{array}{l}\text { Sweden } \\
12.9\end{array}$ & $\begin{array}{l}\text { NLD } \\
10.7\end{array}$ & $\begin{array}{l}\text { CHE } \\
\text { NA }\end{array}$ & $\begin{array}{l}\text { Japan } \\
\text { NA }\end{array}$ & 20.2 \\
\hline
\end{tabular}

NA indicates not applicable. CHE indicates Switzerland; NLD, the Netherlands. See eTable 5 in Supplement 2 for data ordered by country.

a US discounted prices are listed; nondiscounted prices are $\$ 216.00$ for Crestor, $\$ 372.75$ for long-acting insulin, $\$ 309.60$ for Advair, and $\$ 3430.82$ for Humira.

${ }^{\mathrm{b}} \mathrm{A}$ new chemical entity is a compound without any precedent among the regulated and approved drug products.

c Volume is most often the proportion of total prescriptions that were for generic brands. Volumes can be expressed in defined daily doses or as a number of packages/boxes or standard units. Value is most often the proportion of total cost (ie, government and patient expenditure) that was for generic brands. Values can be, for instance, the turnover of pharmaceutical companies, the amount paid for pharmaceuticals by third-party payers, or the amount paid by all payers (third-party and consumers). Market value is most often at ex-factory prices, while amounts paid by third-party payers and consumers are in general at retail prices.

${ }^{d}$ Defined daily dose is the assumed mean maintenance dose per day for a drug used for its main indication in adults. Data shown here are actual mean defined daily doses for each country. the salaries paid to both generalist and specialist physicians were markedly higher in the United States, where specialists were paid twice as much as those in the United Kingdom or Germany and primary care physicians and nurses also had substantially higher salaries. However, it can be difficult to compare salaries of workers from various countries. In general, salaries for professionals are higher in the United States than in other countries, and in addition, workers in the United States bear higher costs in some areas, such as for health care insurance, higher education, or planning for retirement, than workers in other countries. Some of these issues are accounted for in the ratio of national average wage, but US physicians and nurses still had the highest ratio.

Some of the differential observed in cost between physicians and nurses in the United States and in the other countries may reflect differences in productivity or the extent to which training costs are borne by the individual, although in either case it is unlikely to account for the magnitude of the difference. In 2011, Laugesen and Glied $^{19}$ estimated that the investment repayment cost for private education in the United States would amount to about $\$ 21300$ per year for a primary care physician and about $\$ 24400$ for an orthopedic surgeon over a 35-year period.

Taking this investment into account, however, does not explain the more than $\$ 200000$ difference in compensation observed for physicians between countries. Although remuneration varies widely across systems, the optimal level of compensation remains unclear. Salaries in the United States may be high, but recent debates on remuneration of medical staff in the United Kingdom and France, for example, suggest that salaries in other countries may be too low. ${ }^{22}$ Other indicators, such as average wages for competitive nonhealth professions or retention of medical graduates, may help inform appropriate salaries of health care professionals in a given country.

Prices of services were not examined directly, but US health care spending was found to be higher than in other countries despite similar utilization patterns, suggesting that higher prices were the primary cause of high health care spending in the United States relative to other nations. This is consistent with prior work 
Figure 10. Access and Quality

\begin{tabular}{|c|c|c|c|c|c|c|c|c|c|c|c|c|}
\hline Rank (highest to lowest) & 1 & 2 & 3 & 4 & 5 & 6 & 7 & 8 & 9 & 10 & 11 & Mean \\
\hline \multicolumn{13}{|l|}{ Access, $\%$} \\
\hline $\begin{array}{l}\text { Able to get same- or next- } \\
\text { day appointment }{ }^{\mathrm{a}}\end{array}$ & $\begin{array}{l}\text { NLD } \\
77\end{array}$ & $\begin{array}{l}\text { Australia } \\
67\end{array}$ & $\begin{array}{l}\text { UK } \\
57\end{array}$ & $\begin{array}{l}\text { France } \\
56\end{array}$ & $\begin{array}{l}\text { Germany } \\
53\end{array}$ & $\begin{array}{l}\text { US } \\
51\end{array}$ & $\begin{array}{l}\text { Sweden } \\
49\end{array}$ & $\begin{array}{l}\text { Canada } \\
43\end{array}$ & $\begin{array}{l}\text { CHE } \\
\text { NA }\end{array}$ & $\begin{array}{l}\text { Denmark } \\
\text { NA }\end{array}$ & $\begin{array}{l}\text { Japan } \\
\text { NA }\end{array}$ & 57 \\
\hline 2-mo Wait time to see specialist & $\begin{array}{l}\text { Canada } \\
39\end{array}$ & $\begin{array}{l}\text { UK } \\
19\end{array}$ & $\begin{array}{l}\text { Sweden } \\
19\end{array}$ & $\begin{array}{l}\text { Australia } \\
13\end{array}$ & $\begin{array}{l}\text { CHE } \\
9\end{array}$ & $\begin{array}{l}\text { NLD } \\
7\end{array}$ & $\begin{array}{l}\text { US } \\
6\end{array}$ & $\begin{array}{l}\text { France } \\
4\end{array}$ & $\begin{array}{l}\text { Germany } \\
3\end{array}$ & $\begin{array}{l}\text { Denmark } \\
\text { NA }\end{array}$ & $\begin{array}{l}\text { Japan } \\
\text { NA }\end{array}$ & 13 \\
\hline $\begin{array}{l}\text { Adequate time with regular } \\
\text { (primary) physician }\end{array}$ & $\begin{array}{l}\text { Germany } \\
88\end{array}$ & $\begin{array}{l}\text { UK } \\
86\end{array}$ & $\begin{array}{l}\text { NLD } \\
85\end{array}$ & $\begin{array}{l}\text { CHE } \\
84\end{array}$ & $\begin{array}{l}\text { Australia } \\
83\end{array}$ & $\begin{array}{l}\text { US } \\
81\end{array}$ & $\begin{array}{l}\text { Canada } \\
79\end{array}$ & $\begin{array}{l}\text { Sweden } \\
78\end{array}$ & $\begin{array}{l}\text { France } \\
\text { NA }\end{array}$ & $\begin{array}{l}\text { Denmark } \\
\text { NA }\end{array}$ & $\begin{array}{l}\text { Japan } \\
\text { NA }\end{array}$ & 83 \\
\hline \multicolumn{13}{|l|}{ Perceptions, \% } \\
\hline System works well & $\begin{array}{l}\text { Germany } \\
60\end{array}$ & $\begin{array}{l}\text { CHE } \\
58\end{array}$ & $\begin{array}{l}\text { France } \\
54\end{array}$ & $\begin{array}{l}\text { UK } \\
44\end{array}$ & $\begin{array}{l}\text { Sweden } \\
44\end{array}$ & $\begin{array}{l}\text { Australia } \\
44\end{array}$ & $\begin{array}{l}\text { Canada } \\
35\end{array}$ & $\begin{array}{l}\text { US } \\
19\end{array}$ & $\begin{array}{l}\text { NLD } \\
\text { NA }\end{array}$ & $\begin{array}{l}\text { Denmark } \\
\text { NA }\end{array}$ & $\begin{array}{l}\text { Japan } \\
\text { NA }\end{array}$ & 45 \\
\hline Fundamental changes needed & $\begin{array}{l}\text { Canada } \\
55\end{array}$ & $\begin{array}{l}\text { US } \\
53\end{array}$ & $\begin{array}{l}\text { UK } \\
46\end{array}$ & $\begin{array}{l}\text { Sweden } \\
46\end{array}$ & $\begin{array}{l}\text { Australia } \\
46\end{array}$ & $\begin{array}{l}\text { France } \\
41\end{array}$ & $\begin{array}{l}\text { Germany } \\
37\end{array}$ & $\begin{array}{l}\text { CHE } \\
37\end{array}$ & $\begin{array}{l}\text { NLD } \\
\text { NA }\end{array}$ & $\begin{array}{l}\text { Denmark } \\
\text { NA }\end{array}$ & $\begin{array}{l}\text { Japan } \\
\text { NA }\end{array}$ & 45 \\
\hline $\begin{array}{l}\text { Complete rebuild of health } \\
\text { system needed }\end{array}$ & $\begin{array}{l}\text { US } \\
23\end{array}$ & $\begin{array}{l}\text { Sweden } \\
10\end{array}$ & $\begin{array}{l}\text { Canada } \\
9\end{array}$ & $\begin{array}{l}\text { UK } \\
7\end{array}$ & $\begin{array}{l}\text { France } \\
4\end{array}$ & $\begin{array}{l}\text { Australia } \\
4\end{array}$ & $\begin{array}{l}\text { Germany } \\
3\end{array}$ & $\begin{array}{l}\text { CHE } \\
3\end{array}$ & $\begin{array}{l}\text { NLD } \\
\text { NA }\end{array}$ & $\begin{array}{l}\text { Denmark } \\
\text { NA }\end{array}$ & $\begin{array}{l}\text { Japan } \\
\text { NA }\end{array}$ & 8 \\
\hline \multicolumn{13}{|l|}{ Prevention } \\
\hline $\begin{array}{l}\text { Measles immunization, \% } \\
\text { of children }\end{array}$ & $\begin{array}{l}\text { Sweden } \\
98\end{array}$ & $\begin{array}{l}\text { Japan } \\
98\end{array}$ & $\begin{array}{l}\text { Germany } \\
97\end{array}$ & $\begin{array}{l}\text { NLD } \\
96\end{array}$ & $\begin{array}{l}\text { UK } \\
93\end{array}$ & $\begin{array}{l}\text { CHE } \\
93\end{array}$ & $\begin{array}{l}\text { Australia } \\
93\end{array}$ & $\begin{array}{l}\text { US } \\
92\end{array}$ & $\begin{array}{l}\text { France } \\
91\end{array}$ & $\begin{array}{l}\text { Denmark } \\
91\end{array}$ & $\begin{array}{l}\text { Canada } \\
90\end{array}$ & 94 \\
\hline $\begin{array}{l}\text { Breast cancer screening, \% } \\
\text { of women aged } 50-69 \mathrm{y}^{\mathrm{b}}\end{array}$ & $\begin{array}{l}\text { Denmark } \\
84\end{array}$ & $\begin{array}{l}\text { US } \\
81\end{array}$ & $\begin{array}{l}\text { NLD } \\
79\end{array}$ & $\begin{array}{l}\text { UK } \\
76\end{array}$ & $\begin{array}{l}\text { Sweden } \\
75\end{array}$ & $\begin{array}{l}\text { Canada } \\
72\end{array}$ & $\begin{array}{l}\text { Germany } \\
71\end{array}$ & $\begin{array}{l}\text { Australia } \\
55\end{array}$ & $\begin{array}{l}\text { France } \\
52\end{array}$ & $\begin{array}{l}\text { CHE } \\
47\end{array}$ & $\begin{array}{l}\text { Japan } \\
41\end{array}$ & 67 \\
\hline \multicolumn{13}{|l|}{ Clinical outcomes } \\
\hline $\begin{array}{l}\text { 30-d Stroke mortality per } \\
1000 \text { patients }^{c}\end{array}$ & $\begin{array}{l}\text { Canada } \\
10\end{array}$ & $\begin{array}{l}\text { Sweden } \\
9.6\end{array}$ & $\begin{array}{l}\text { Australia } \\
9.3\end{array}$ & $\begin{array}{l}\text { UK } \\
9.2\end{array}$ & $\begin{array}{l}\text { France } \\
7.9\end{array}$ & $\begin{array}{l}\text { CHE } \\
6.9\end{array}$ & $\begin{array}{l}\text { Germany } \\
6.4\end{array}$ & $\begin{array}{l}\text { US } \\
4.2\end{array}$ & $\begin{array}{l}\text { NLD } \\
\text { NA }\end{array}$ & $\begin{array}{l}\text { Denmark } \\
\text { NA }\end{array}$ & $\begin{array}{l}\text { Japan } \\
\text { NA }\end{array}$ & 7.9 \\
\hline $\begin{array}{l}30-d \text { Mortality per } 1000 \text { patients } \\
\text { with acute myocardial infarction }\end{array}$ & $\begin{array}{l}\text { Germany } \\
8.7\end{array}$ & $\begin{array}{l}\text { Sweden } \\
8.3\end{array}$ & $\begin{array}{l}\text { CHE } \\
7.7\end{array}$ & $\begin{array}{l}\text { UK } \\
7.6\end{array}$ & $\begin{array}{l}\text { France } \\
7.2\end{array}$ & $\begin{array}{l}\text { Canada } \\
6.7\end{array}$ & $\begin{array}{l}\text { US } \\
5.5\end{array}$ & $\begin{array}{l}\text { Australia } \\
4.1\end{array}$ & $\begin{array}{l}\text { NLD } \\
\text { NA }\end{array}$ & $\begin{array}{l}\text { Denmark } \\
\text { NA }\end{array}$ & $\begin{array}{l}\text { Japan } \\
\text { NA }\end{array}$ & 7 \\
\hline $\begin{array}{l}\text { Foreign body left per } \\
100000 \text { discharges }\end{array}$ & $\begin{array}{l}\text { CHE } \\
12.3\end{array}$ & $\begin{array}{l}\text { Canada } \\
8.6\end{array}$ & $\begin{array}{l}\text { Australia } \\
8.6\end{array}$ & $\begin{array}{l}\text { France } \\
6.2\end{array}$ & $\begin{array}{l}\text { UK } \\
6.1\end{array}$ & $\begin{array}{l}\text { Germany } \\
5.5\end{array}$ & $\begin{array}{l}\text { Sweden } \\
4.6\end{array}$ & $\begin{array}{l}\text { US } \\
4.1\end{array}$ & $\begin{array}{l}\text { NLD } \\
\text { NA }\end{array}$ & $\begin{array}{l}\text { Denmark } \\
\text { NA }\end{array}$ & $\begin{array}{l}\text { Japan } \\
\text { NA }\end{array}$ & 7 \\
\hline $\begin{array}{l}\text { Obstetric trauma without } \\
\text { instrument per } 100 \text { deliveries }\end{array}$ & $\begin{array}{l}\text { Canada } \\
3.1 \\
\end{array}$ & $\begin{array}{l}\text { UK } \\
2.8\end{array}$ & $\begin{array}{l}\text { Sweden } \\
2.8\end{array}$ & $\begin{array}{l}\text { CHE } \\
2.6\end{array}$ & $\begin{array}{l}\text { Denmark } \\
2.6\end{array}$ & $\begin{array}{l}\text { NLD } \\
2.5\end{array}$ & $\begin{array}{l}\text { Australia } \\
2.4\end{array}$ & $\begin{array}{l}\text { Germany } \\
2.1\end{array}$ & $\begin{array}{l}\text { US } \\
1.5\end{array}$ & $\begin{array}{l}\text { France } \\
0.6\end{array}$ & $\begin{array}{l}\text { Japan } \\
\text { NA }\end{array}$ & 2.3 \\
\hline \multicolumn{13}{|l|}{ Avoidable hospitalizations } \\
\hline $\begin{array}{l}\text { Diabetes hospitalizations per } \\
100000 \text { population }^{d}\end{array}$ & $\begin{array}{l}\text { Germany } \\
218.3\end{array}$ & $\begin{array}{l}\text { US } \\
191\end{array}$ & $\begin{array}{l}\text { Japan } \\
162.3\end{array}$ & $\begin{array}{l}\text { France } \\
150.6\end{array}$ & $\begin{array}{l}\text { Australia } \\
141.1\end{array}$ & $\begin{array}{l}\text { Denmark } \\
113.4\end{array}$ & $\begin{array}{l}\text { Sweden } \\
96\end{array}$ & $\begin{array}{l}\text { Canada } \\
93.7\end{array}$ & $\begin{array}{l}\text { UK } \\
72.8\end{array}$ & $\begin{array}{l}\text { CHE } \\
72.6\end{array}$ & $\begin{array}{l}\text { NLD } \\
69.8\end{array}$ & 125.6 \\
\hline $\begin{array}{l}\text { Diabetes hospitalizations as a } \\
\text { ratio of population with diabetes }\end{array}$ & $\begin{array}{l}\text { Japan } \\
2.80\end{array}$ & $\begin{array}{l}\text { Australia } \\
2.80\end{array}$ & $\begin{array}{l}\text { Germany } \\
2.40\end{array}$ & $\begin{array}{l}\text { US } \\
2\end{array}$ & $\begin{array}{l}\text { Sweden } \\
1.90\end{array}$ & $\begin{array}{l}\text { Denmark } \\
1.80\end{array}$ & $\begin{array}{l}\text { UK } \\
1.70\end{array}$ & $\begin{array}{l}\text { Canada } \\
1.30\end{array}$ & $\begin{array}{l}\text { France } \\
1.20\end{array}$ & $\begin{array}{l}\text { NLD } \\
1.20\end{array}$ & $\begin{array}{l}\text { CHE } \\
1.20\end{array}$ & 2.00 \\
\hline $\begin{array}{l}\text { Asthma hospitalizations per } \\
100000 \text { population }^{f}\end{array}$ & $\begin{array}{l}\text { US } \\
89.7\end{array}$ & $\begin{array}{l}\text { UK } \\
71\end{array}$ & $\begin{array}{l}\text { Australia } \\
64.8\end{array}$ & $\begin{array}{l}\text { Denmark } \\
50.6\end{array}$ & $\begin{array}{l}\text { NLD } \\
36\end{array}$ & $\begin{array}{l}\text { Japan } \\
34.7\end{array}$ & $\begin{array}{l}\text { France } \\
29.6\end{array}$ & $\begin{array}{l}\text { Germany } \\
28.7\end{array}$ & $\begin{array}{l}\text { CHE } \\
27.5\end{array}$ & $\begin{array}{l}\text { Sweden } \\
19\end{array}$ & $\begin{array}{l}\text { Canada } \\
14.6\end{array}$ & 42.4 \\
\hline $\begin{array}{l}\text { Asthma hospitalizations as a } \\
\text { ratio of population with asthmag }\end{array}$ & $\begin{array}{l}\text { US } \\
1.20\end{array}$ & $\begin{array}{l}\text { UK } \\
1.00\end{array}$ & $\begin{array}{l}\text { France } \\
0.80\end{array}$ & $\begin{array}{l}\text { Denmark } \\
0.80\end{array}$ & $\begin{array}{l}\text { Germany } \\
0.70\end{array}$ & $\begin{array}{l}\text { NLD } \\
0.70\end{array}$ & $\begin{array}{l}\text { Australia } \\
0.60\end{array}$ & $\begin{array}{l}\text { CHE } \\
0.40\end{array}$ & $\begin{array}{l}\text { Sweden } \\
0.30\end{array}$ & $\begin{array}{l}\text { Japan } \\
0.30\end{array}$ & $\begin{array}{l}\text { Canada } \\
0.20\end{array}$ & 0.70 \\
\hline
\end{tabular}

NA indicates not applicable. CHE indicates Switzerland; NLD, the Netherlands. See eTable 6 in Supplement 2 for data ordered by country.

a Able to get same- or next-day appointment when sick, excluding those who did not need to see a physician or nurse.

${ }^{\mathrm{b}}$ Women aged 40 to 49 years in Sweden.

c Thirty-day stroke mortality after hospital admission for ischemic stroke.
${ }^{\mathrm{d}}$ Limited to a primary diagnosis of diabetes.

e Diabetes hospitalizations are limited to persons aged 15 years or older, whereas the denominator (population with diabetes) is both adult and pediatric.

${ }^{f}$ Limited to a primary diagnosis of asthma.

${ }^{g}$ Asthma hospitalizations are limited to persons aged 15 years or older, whereas the denominator (population with asthma) is both adult and pediatric. by Anderson et al, ${ }^{23}$ which also illustrated higher health care spending in the United States despite similar inputs and levels of care utilization, and more recent work by Dieleman et al..$^{13}$ In addition to differential prices for physician services and pharmaceuticals, these results suggest that prices for nonphysician services and procedures also appeared to be markedly higher in the United States. For the select procedures for which comparable data were available, the United States paid considerably more than its counterparts. In 2013, the International Federation of Health Plans ${ }^{24}$ reported that the average cost in the United States was $\$ 75345$ for a coronary artery bypass graft surgery, whereas the costs in the Netherlands and Switzerland were \$15 742 and \$36 509, respectively. Computed tomography was also much higher in the
United States, with an average payment of $\$ 896$ per scan compared with $\$ 97$ in Canada, $\$ 279$ in the Netherlands, \$432 in Switzerland, and $\$ 500$ in Australia in 2013. Similarly, the mean payment for an MRI in the United States was $\$ 1145$ compared with $\$ 350$ in Australia and $\$ 461$ in the Netherlands.

Of particular interest to US health policy makers is the role of the pharmaceutical market and its influence on health care spending. Not surprisingly, US spending on pharmaceuticals was almost double the spending in comparison countries. Previous work suggests that this is driven by high prices for brand-name drugs rather than by utilization, which is comparable with other high-income countries. ${ }^{24-27}$ Across comparison countries, the United States had the highest volume of generics, accounting for more than $80 \%$ of 
Figure 11. Distribution and Equity

\begin{tabular}{|c|c|c|c|c|c|c|c|c|c|c|c|c|}
\hline Rank (highest to lowest) & 1 & 2 & 3 & 4 & 5 & 6 & 7 & 8 & 9 & 10 & 11 & Mean \\
\hline \multicolumn{13}{|l|}{ Equity } \\
\hline Horizontal inequity index, \%a & $\begin{array}{l}\text { US } \\
6\end{array}$ & $\begin{array}{l}\text { Canada } \\
1.90\end{array}$ & $\begin{array}{l}\text { France } \\
1.30\end{array}$ & $\begin{array}{l}\text { Germany } \\
1.00\end{array}$ & $\begin{array}{l}\text { UK } \\
0.40\end{array}$ & $\begin{array}{l}\text { Sweden } \\
\text { NA }\end{array}$ & $\begin{array}{l}\text { NLD } \\
\text { NA }\end{array}$ & $\begin{array}{l}\text { CHE } \\
\text { NA }\end{array}$ & $\begin{array}{l}\text { Denmark } \\
\text { NA }\end{array}$ & $\begin{array}{l}\text { Japan } \\
\text { NA }\end{array}$ & $\begin{array}{l}\text { Australia } \\
\text { NA }\end{array}$ & 2.10 \\
\hline \multicolumn{13}{|l|}{ Out-of-pocket spending } \\
\hline As $\%$ of total health expenditure & $\begin{array}{l}\text { CHE } \\
26.8\end{array}$ & $\begin{array}{l}\text { Australia } \\
18.8\end{array}$ & $\begin{array}{l}\text { Sweden } \\
14.1\end{array}$ & $\begin{array}{l}\text { Japan } \\
13.9\end{array}$ & $\begin{array}{l}\text { Canada } \\
13.6\end{array}$ & $\begin{array}{l}\text { Denmark } \\
13.4\end{array}$ & $\begin{array}{l}\text { Germany } \\
13.2\end{array}$ & $\begin{array}{l}\text { US } \\
11\end{array}$ & $\begin{array}{l}\text { UK } \\
9.7\end{array}$ & $\begin{array}{l}\text { France } \\
6.3\end{array}$ & $\begin{array}{l}\text { NLD } \\
5.2\end{array}$ & 13.3 \\
\hline As \% of household consumption & $\begin{array}{l}\text { CHE } \\
4.5\end{array}$ & $\begin{array}{l}\text { Sweden } \\
3.4\end{array}$ & $\begin{array}{l}\text { Australia } \\
3.2\end{array}$ & $\begin{array}{l}\text { US } \\
2.6\end{array}$ & $\begin{array}{l}\text { Denmark } \\
2.6\end{array}$ & $\begin{array}{l}\text { Canada } \\
2.3\end{array}$ & $\begin{array}{l}\text { Japan } \\
2.2\end{array}$ & $\begin{array}{l}\text { Germany } \\
1.8\end{array}$ & $\begin{array}{l}\text { UK } \\
1.4\end{array}$ & $\begin{array}{l}\text { France } \\
1.4\end{array}$ & $\begin{array}{l}\text { NLD } \\
1.3\end{array}$ & 2.4 \\
\hline \multicolumn{13}{|l|}{ Unmet need $^{b}$} \\
\hline $\begin{array}{l}\text { Consultation skipped } \\
\text { because of cost }\end{array}$ & $\begin{array}{l}\text { US } \\
22.3\end{array}$ & $\begin{array}{l}\text { Australia } \\
16.2\end{array}$ & $\begin{array}{l}\text { NLD } \\
12.5\end{array}$ & $\begin{array}{l}\text { France } \\
9\end{array}$ & $\begin{array}{l}\text { CHE } \\
7\end{array}$ & $\begin{array}{l}\text { Canada } \\
6.6\end{array}$ & $\begin{array}{l}\text { UK } \\
4.2\end{array}$ & $\begin{array}{l}\text { Sweden } \\
3.9\end{array}$ & $\begin{array}{l}\text { Germany } \\
2.6\end{array}$ & $\begin{array}{l}\text { Denmark } \\
\text { NA }\end{array}$ & $\begin{array}{l}\text { Japan } \\
\text { NA }\end{array}$ & 9.4 \\
\hline $\begin{array}{l}\% \text { Unmet need, below-average } \\
\text { income }\end{array}$ & $\begin{array}{l}\text { US } \\
43\end{array}$ & $\begin{array}{l}\text { CHE } \\
31\end{array}$ & $\begin{array}{l}\text { France } \\
30\end{array}$ & $\begin{array}{l}\text { Canada } \\
30\end{array}$ & $\begin{array}{l}\text { NLD } \\
29\end{array}$ & $\begin{array}{l}\text { Australia } \\
24\end{array}$ & $\begin{array}{l}\text { Germany } \\
16\end{array}$ & $\begin{array}{l}\text { Sweden } \\
16\end{array}$ & $\begin{array}{l}\text { UK } \\
8\end{array}$ & $\begin{array}{l}\text { Denmark } \\
\text { NA }\end{array}$ & $\begin{array}{l}\text { Japan } \\
\text { NA }\end{array}$ & 25.2 \\
\hline $\begin{array}{l}\% \text { Unmet need, above-average } \\
\text { income }\end{array}$ & $\begin{array}{l}\text { US } \\
32\end{array}$ & $\begin{array}{l}\text { CHE } \\
22\end{array}$ & $\begin{array}{l}\text { NLD } \\
16\end{array}$ & $\begin{array}{l}\text { France } \\
14\end{array}$ & $\begin{array}{l}\text { Canada } \\
13\end{array}$ & $\begin{array}{l}\text { Australia } \\
13\end{array}$ & $\begin{array}{l}\text { UK } \\
7\end{array}$ & $\begin{array}{l}\text { Sweden } \\
7\end{array}$ & $\begin{array}{l}\text { Germany } \\
6\end{array}$ & $\begin{array}{l}\text { Denmark } \\
\text { NA }\end{array}$ & $\begin{array}{l}\text { Japan } \\
\text { NA }\end{array}$ & 14.4 \\
\hline \multicolumn{13}{|l|}{ Geographic breakdown } \\
\hline $\begin{array}{l}\text { Rural population, } \% \text { of } \\
\text { total population }\end{array}$ & $\begin{array}{l}\text { CHE } \\
26\end{array}$ & $\begin{array}{l}\text { France } \\
20\end{array}$ & $\begin{array}{l}\text { US } \\
18\end{array}$ & $\begin{array}{l}\text { Germany } \\
18\end{array}$ & $\begin{array}{l}\text { Canada } \\
18\end{array}$ & $\begin{array}{l}\text { UK } \\
17\end{array}$ & $\begin{array}{l}\text { Sweden } \\
14\end{array}$ & $\begin{array}{l}\text { Denmark } \\
12\end{array}$ & $\begin{array}{l}\text { Australia } \\
10\end{array}$ & $\begin{array}{l}\text { NLD } \\
9\end{array}$ & $\begin{array}{l}\text { Japan } \\
6\end{array}$ & 15 \\
\hline Population density per sq mile & $\begin{array}{l}\text { NLD } \\
505\end{array}$ & $\begin{array}{l}\text { Japan } \\
348\end{array}$ & $\begin{array}{l}\text { UK } \\
271\end{array}$ & $\begin{array}{l}\text { Germany } \\
237\end{array}$ & $\begin{array}{l}\text { CHE } \\
212\end{array}$ & $\begin{array}{l}\text { Denmark } \\
136\end{array}$ & $\begin{array}{l}\text { France } \\
122\end{array}$ & $\begin{array}{l}\text { US } \\
35\end{array}$ & $\begin{array}{l}\text { Sweden } \\
24\end{array}$ & $\begin{array}{l}\text { Canada } \\
4\end{array}$ & $\begin{array}{l}\text { Australia } \\
3\end{array}$ & 173 \\
\hline $\begin{array}{l}\text { Urban physicians per } \\
1000 \text { population }\end{array}$ & $\begin{array}{l}\text { Sweden } \\
4.5\end{array}$ & $\begin{array}{l}\text { France } \\
4.1\end{array}$ & $\begin{array}{l}\text { Canada } \\
4.1\end{array}$ & $\begin{array}{l}\text { US } \\
3.2\end{array}$ & $\begin{array}{l}\text { Japan } \\
2.9\end{array}$ & $\begin{array}{l}\text { CHE } \\
2.8\end{array}$ & $\begin{array}{l}\text { Australia } \\
2.6\end{array}$ & $\begin{array}{l}\text { Germany } \\
2\end{array}$ & $\begin{array}{l}\text { UK } \\
\text { NA }\end{array}$ & $\begin{array}{l}\text { NLD } \\
\text { NA }\end{array}$ & $\begin{array}{l}\text { Denmark } \\
\text { NA }\end{array}$ & 3.3 \\
\hline $\begin{array}{l}\text { Rural physicians per } \\
1000 \text { population }\end{array}$ & $\begin{array}{l}\text { CHE } \\
4.4\end{array}$ & $\begin{array}{l}\text { Sweden } \\
3.5\end{array}$ & $\begin{array}{l}\text { France } \\
2.5\end{array}$ & $\begin{array}{l}\text { Australia } \\
1.7\end{array}$ & $\begin{array}{l}\text { US } \\
1.4\end{array}$ & $\begin{array}{l}\text { Japan } \\
1.4\end{array}$ & $\begin{array}{l}\text { Germany } \\
1.3\end{array}$ & $\begin{array}{l}\text { Canada } \\
0.4\end{array}$ & $\begin{array}{l}\text { UK } \\
\text { NA }\end{array}$ & $\begin{array}{l}\text { NLD } \\
\text { NA }\end{array}$ & $\begin{array}{l}\text { Denmark } \\
\text { NA }\end{array}$ & 2.1 \\
\hline
\end{tabular}

NA indicates not applicable. CHE indicates Switzerland; NLD, the Netherlands. See eTable 7 in Supplement 2 for data ordered by country.

${ }^{a}$ The horizontal inequity index is the percentage probability of a physician visit in the past 12 months by wealth. If the index is greater than 0 , then high-income groups access physicians more than low-income groups after adjustment for relative need.

${ }^{\mathrm{b}}$ Regarding unmet need, the Commonwealth Fund International Health Policy
Survey poses a number of questions to a representative sample from each country. For this indicator, it was analyzed whether a respondent did not consult with or visit a physician because of cost, skipped a medical test, treatment, or follow-up that was recommended by a physician because of cost, or did not fill or collect a prescription for medicine or skipped doses of medicine because of cost. Low income is defined as household income less than $50 \%$ of the country median. total pharmaceuticals used by US residents yet just under $30 \%$ of the United States' total spending on pharmaceuticals. Australia and France had much smaller generic market penetration rates, presumably because brand-name drugs are comparatively affordable. ${ }^{25}$ Although the United States' high prices of pharmaceuticals are controversial, these prices have been viewed as critical to innovation, including US production of new chemical entities. ${ }^{18,28}$ Whether innovation justifies high levels of spending is not clear.

Performance on access is also a central concern to policy makers. These data indicated that the United States had the lowest rate of insurance coverage. However, the percentage of the population with health insurance is at a historical high and has continued to increase since the passage of the Affordable Care Act. Out-of-pocket spending was also surprisingly low in the United States, which may be explained in part by the relatively high proportion of patients who do not seek care or who skip consultations because of high costs. Prior work has shown that out-of-pocket spending is more concentrated among the poor. ${ }^{29}$ Other factors influencing access to care in the United States aside from affordability likely include the country's considerable landmass (and correspondingly large rural population). Personal choice is an additional albeit contentious factor. For example, the Kaiser Family Foundation has found that approximately $20 \%$ of uninsured US residents have incomes of $400 \%$ or more of the federal poverty level and have largely chosen to forgo insurance. ${ }^{30}$ In the other countries in this study with similar health insurance designs (the Netherlands and Switzerland), the proportion of the population choosing to forego coverage is considerably less. ${ }^{31,32}$ Still, a substantial proportion of people would benefit from coverage but remain uninsured in the United States, and increasing coverage for these individuals remains a policy priority.

A central concern in the United States is the extent to which greater health care spending translates to better outcomes. Comparing intermediate country-level health care outcomes across a range of health care services showed that the United States does perform favorably on certain acute care outcomes but less so for primary care measures. Notably, the United States had among the highest breast cancer screening rates and the lowest 30-day mortality rates for acute myocardial infarction and stroke. Although the rates of avoidable hospitalizations in the United States, such as for diabetes and asthma, are well above those of most of the countries in the analysis, accounting for the United States' higher prevalence of both diseases reduced this gap considerably. The United States has relatively poor population health outcomes, which likely represents a combination of factors including issues with the affordability of care. However, the United States average, in comparison to averages of much smaller, more homogeneous countries, may lead to erroneous conclusions. For example, the life expectancy of Minnesota, a state comparable in size and demographics to 
Sweden or Denmark, has more similar population health outcomes to these countries than Minnesota has in comparison to Mississippi.

This analysis extends to a body of work that has explored US health care spending ${ }^{33}$ by revisiting comparisons following the passage of the Affordable Care Act, which has both increased coverage and spurred health care delivery reform toward the delivery of high-value care. ${ }^{9,13}$ Similar to previous work by Garber and Skinner, ${ }^{10}$ Reinhardt et al, ${ }_{12}^{12}$ and Anderson et $\mathrm{al}^{23}$ that examined US health care spending relative to other OECD countries, we found that the United States spent significantly more on health care despite having similar levels of utilization. However, from 2010 onward it appeared that the United States had similar levels of public social spending compared with other high-income countries. While public social spending has increased since 2010 (from 15.6\% of GDP in 2005 to $19.3 \%$ of GDP in 2010), utilization rates have remained relatively unchanged. ${ }^{20,23}$ This finding suggests that differences in social spending are less likely to be the main driver of differences in health care spending than previous works have suggested. ${ }^{34}$ This is a similar conclusion to recent work by Dieleman et $\mathrm{al}^{13}{ }^{13}$ which identified prices and intensity of care to be largely related to increases in health care spending over the past 15 years and negatively associated with disease prevalence or incidence.

This study has several limitations. First, there was limited availability of comparable data across health systems, which restricted the areas of health system performance we were able to compare. In particular, data on prices across systems were lacking, as was the number of indicators on quality and coverage. The indicators selected thus provide a snapshot of performance in some domainsparticularly quality-which may not be representative of quality of the entire system or may be limited due to data availability across all countries. For instance, 30-day mortality rates reflect only inhospital rates, which may influence the apparent variations observed, particularly given differences in length of stay and discharge practices across countries. Second, because of the difficulties in collecting and standardizing indicators across countries, some of the data used reflect different years, may be out of date, or may have been measured differently based on country-specific definitions of variables. Even when data were collected from the same source, issues of comparability remain because of fundamental differences in how systems are organized and, in turn, how care is categorized. Two areas of particular concern are outpatient spending and the primary care workforce. We attempted to address limitations in the workforce data by utilizing a functionality-based approach to identifying who provides primary care services in each country and by cross-referencing resulting numbers with country experts. Third, the study was able to present only descriptive comparative findings, and it is not possible to make any causal inferences. Fourth, the data did not adjust for factors such as underlying population differences or system delivery and organizational factors, which likely influence some of the observed variation in this space. Fifth, the response rate to some surveys that were the basis for some measures, such as ability to get same- or next-day appointment and 2-month wait time to see a specialist physician, were not good across all countries. Sixth, we did not consider the actual prices of devices, which, given the increasing number of hip and knee replacements and use of other devices, are emerging as an important consideration in the cost of care in the United States.

\section{Conclusions}

The United States spent approximately twice as much as other high-income countries on medical care, yet utilization rates in the United States were largely similar to those in other nations. Prices of labor and goods, including pharmaceuticals, and administrative costs appeared to be the major drivers of the difference in overall cost between the United States and other high-income countries. As patients, physicians, policy makers, and legislators actively debate the future of the US health system, data such as these are needed to inform policy decisions.

\section{ARTICLE INFORMATION}

Accepted for Publication: February 2, 2018.

Author Contributions: Dr Papanicolas and Ms Woskie had full access to all of the data in the study and take responsibility for the integrity of the data and the accuracy of the data analysis.

Concept and design: All authors.

Acquisition, analysis, or interpretation of data: All authors.

Drafting of the manuscript: Papanicolas, Woskie. Critical revision of the manuscript for important intellectual content: All authors.

Statistical analysis: Woskie.

Administrative, technical, or material support: All authors.

Supervision: Papanicolas, Jha.

Conflict of Interest Disclosures: All authors have completed and submitted the ICMJE Form for Disclosure of Potential Conflicts of Interest and none were reported.

Additional Contributions: We thank Duncan Orlander, BA, Harvard School of Public Health Department of Health Policy and Management, for research assistance (compensated as part of regular pay).

\section{REFERENCES}

1. World Health Organization. Global Health Expenditure Database. http://apps.who.int/nha /database/Country_Profile/Index/en. Accessed September 22, 2017.

2. Health at a Glance 2017. Paris, France: OECD Publishing; 2017. doi:10.1787/health_glance -2017-en.

3. Sorensen N, Reed JD, Bollyky T, et al; GBD 2015 Healthcare Access and Quality Collaborators. Healthcare Access and Quality Index based on mortality from causes amenable to personal health care in 195 countries and territories, 1990-2015: a novel analysis from the Global Burden of Disease Study 2015. Lancet. 2017;390(10091):231-266.

4. Schneider EC, Sarnak DO, Squires D, Shah A, Doty MM. Mirror, Mirror 2017: International Comparison Reflects Flaws and Opportunities for Better US Health Care. July 2017.

http://www.commonwealthfund.org/ /media /files/publications/fund-report/2017/jul /schneider_mirror_mirror_2017.pdf. Accessed September 22, 2017.

5. World Health Organization. The World Health Report 2000: Health Systems, Improving
Performance. 2000. http://www.who.int/entity /whr/2000/en/whrO0_en.pdf?ua=1. Accessed January 17, 2018.

6. Obama B. United States health care reform: progress to date and next steps. JAMA. 2016;316 (5):525-532.

7. Schroeder SA, Frist W; National Commission on Physician Payment Reform. Phasing out fee-for-service payment. N Engl J Med. 2013;368 (21):2029-2032.

8. For the Public's Health: Investing in a Healthier Future: Health and Medicine Division. April 10, 2012 http://www.nationalacademies.org/hmd/Reports /2012/For-the-Publics-Health-Investing-in-a -Healthier-Future.aspx. Accessed January 17, 2018.

9. Berwick DM, Hackbarth AD. Eliminating waste in US health care. JAMA. 2012;307(14):1513-1516.

10. Garber AM, Skinner J. Is American health care uniquely inefficient? J Econ Perspect. 2008;22(4): 27-50.

11. Bradley EH, Elkins BR, Herrin J, Elbel B. Health and social services expenditures: associations with health outcomes. BMJ Qual Saf. 2011;20(10):826-831. 
12. Reinhardt UE, Hussey PS, Anderson GFUS US health care spending in an international context. Health Aff (Millwood). 2004;23(3):10-25.

13. Dieleman JL, Squires E, Bui AL, et al. Factors associated with increases in us health care spending, 1996-2013. JAMA. 2017;318(17):1668-1678.

14. Farrell $D$, Jensen $E$, Kocher $B$, et al. Accounting for the Cost of US Health Care: A New Look at Why Americans Spend More. December 2008. https: //www.mckinsey.com/industries/healthcare -systems-and-services/our-insights/accounting -for-the-cost-of-us-health-care. Accessed October 16, 2017.

15. Mossialos E, Djordjevic A, Osborn R, Sarnak D. International Profiles of Health Care Systems: Australia, Canada, China, Denmark, England, France, Germany, India, Israel, Italy, Japan, the Netherlands, New Zealand, Norway, Singapore, Sweden, Switzerland, Taiwan, and the United States. May 31, 2017. http://www.commonwealthfund.org/publications /fund-reports/2017/may/international-profiles. Accessed September 26, 2017.

16. Cylus J, Papanicolas I, Smith PC, eds. Health System Efficiency: How to Make Measurement Matter for Policy and Management. Copenhagen, Denmark: European Observatory on Health Systems and Policies; 2016. Health Policy Series No. 46. http://www.euro.who.int/_data/assets/pdf _file/0004/324283/Health-System-Efficiency -How-make-measurement-matter-policy -management.pdf. Accessed January 20, 2018.

17. Murray CJL, Frenk J. A framework for assessing the performance of health systems. Bull World Health Organ. 2000;78(6):717-731.

18. Daemmrich A. Where Is the Pharmacy to the World? International Regulatory Variation and Pharmaceutical Industry Location. 2009. http://www.hbs.edu/faculty/Publication\%20Files /09-118.pdf. Accessed November 1, 2017.
19. Laugesen MJ, Glied SA. Higher fees paid to US physicians drive higher spending for physician services compared to other countries. Health Aff (Millwood). 2011;30(9):1647-1656.

20. Organisation for Economic Co-operation and Development. Social expenditure database. http://www.oecd.org/social/expenditure.htm. Accessed January 31, 2018.

21. Rechel B, Dubois C-A, Mckee M. The Health Care Workforce in Europe: Learning From Experience. http://www.euro.who.int/_data/assets/pdf _file/0008/91475/E89156.pdf. Accessed November 8, 2017.

22. General Medical Council. The State of Medical Education and Practice in the UK. 2016. https://www.gmc-uk.org/SOMEP_2016_Full _Report_Lo_Res.pdf_68139324.pdf. Accessed November 8, 2017.

23. Anderson GF, Reinhardt UE, Hussey PS, Petrosyan V. It's the prices, stupid: why the United States is so different from other countries. Health Aff (Millwood). 2003;22(3):89-105.

24. International Federation of Health Plans. 2013 Comparative Price Report: Variation in Medical and Hospital Prices by Country. 2013. https://static1.squarespace.com /static/518a3cfee4b0a77d03a62c98/t /534fc9ebe4b05a88e5fbab70/1397737963288 /2013+iFHP+FINAL+4+14+14.pdf. Accessed November 8, 2017.

25. Wouters OJ, Kanavos PG, McKee M. Comparing generic drug markets in Europe and the United States: prices, volumes, and spending. Milbank $Q$. 2017;95(3):554-601.

26. Sarnak DO, Squires D, Kuzmak G, Bishop S. Paying for Prescription Drugs Around the World: Why Is the US an Outlier? October 2017. http://www .commonwealthfund.org/publications/issue-briefs /2017/oct/prescription-drug-costs -us-outlier. Accessed January 21, 2018.

27. O'Neill P, Sussex J. International Comparison of Medicines Usage: Quantitative Analysis. https://www.lif.se/contentassets /a0030c971ca6400e9fbf09a61235263f /international-comparison-of-medicines -usage-quantitative-analysis.pdf. Accessed January 21, 2018.

28. Grabowski HG, Wang YR. The quantity and quality of worldwide new drug introductions, 1982-2003. Health Aff (Millwood). 2006;25(2):452460.

29. Waters HR, Anderson GF, Mays J. Measuring financial protection in health in the United States. Health Policy. 2004;69(3):339-349.

30. Key facts about the uninsured population. September 19, 2017. https://www.kff.org/uninsured /fact-sheet/key-facts-about-the-uninsured -population/. Accessed November 8, 2017.

31. van Ginneken E, Rice T. Enforcing enrollment in health insurance exchanges: evidence from the Netherlands, Switzerland, and Germany. Med Care Res Rev. 2015;72(4):496-509.

32. Oroma N. Administrative efficiency, clearinghouse exchanges, rate review, and zero profits to limit healthcare insurance premium increases. Health Syst Policy Res. 2016;3(4). doi:10.21767/2254-9137.100054

33. French E, Kelly E. Medical spending around the developed world. Fisc Stud. 2016;37(3-4):327-344. doi:10.1111/j.1475-5890.2016.12127

34. Bradley E, Taylor L. The American Health Care Paradox: Why Spending More Is Getting Us Less. 2013. https://yaleglobal.yale.edu/american-health -care-paradox-why-spending-more-getting-us-less. Accessed January 17, 2018. 